\title{
Identification and expression analysis of strigolactone biosynthetic and signaling genes reveal strigolactones are involved in fruit development of the woodland strawberry (Fragaria vesca)
}

Han Wu ${ }^{1 *+} \mathbb{D}$, Huihui $\mathrm{Li}^{1,5^{\dagger}}$, Hong Chen ${ }^{2}$, Qi Qi ${ }^{3}$, Qiangqiang Ding ${ }^{1}$, Juan Xue ${ }^{1}$, Jing Ding ${ }^{1}$, Xiangning Jiang ${ }^{3}$, Xilin $\mathrm{HOu}^{1}$ and $\mathrm{Yi} \mathrm{Li}^{1,4^{*}}$

\begin{abstract}
Background: The development and ripening of fresh fruits is an important trait for agricultural production and fundamental research. Almost all plant hormones participate in this process. Strigolactones (SLs) are a new class of plant hormones that regulate plant organ development and stress tolerance, but little is known about their roles in fruit development.

Results: In this study, we identified SL biosynthetic and signaling genes in woodland strawberry, a typical nonclimacteric fruit, and analyzed the expression patterns of these genes in different plant tissues and developing fruits. One D27, two MAX1, and one $L B O$ gene were identified as involved in SL biosynthesis, and one D14, one $D 3$, and two D53 genes as related to SL signaling. The proteins encoded by these genes had similar motifs as SL biosynthetic and signaling proteins in rice and Arabidopsis. The genes had different expression levels in the root, stem, leaf, and petiole of woodland strawberry. In addition, the expression of most SL biosynthetic genes was high in developing carpel, anther, and style, while that of SL signaling genes was high in carpel and style, but low in anther, suggesting active SL biosynthesis and signaling in the developing carpel and style. Notably, the expression of SL biosynthetic and signaling genes was significantly increased in the receptacle after pollination and decreased during receptacle development. Moreover, low or no expression of these genes was detected in ripening fruits.
\end{abstract}

Conclusions: Our results suggest that SLs play a role in the early stages of woodland strawberry fruit development. Our findings provide insight into the function of SLS and will facilitate further study of the regulation by SLs of fresh fruit development.

Keywords: Woodland strawberry, Genome-wide identification, Expression analysis, Biosynthetic and signaling genes, Strigolactones, Fruit development

\footnotetext{
* Correspondence: wuhan@njau.edu.cn; yilil@uconn.edu

${ }^{\dagger} \mathrm{Han}$ Wu and Huihui Li contributed equally to this work.

${ }^{1}$ State Key Laboratory of Crop Genetics and Germplasm Enhancement,

College of Horticulture, Nanjing Agricultural University, Nanjing 210095,

China

Full list of author information is available at the end of the article
}

(c) The Author(s). 2019 Open Access This article is distributed under the terms of the Creative Commons Attribution 4.0 International License (http://creativecommons.org/licenses/by/4.0/), which permits unrestricted use, distribution, and reproduction in any medium, provided you give appropriate credit to the original author(s) and the source, provide a link to the Creative Commons license, and indicate if changes were made. The Creative Commons Public Domain Dedication waiver (http://creativecommons.org/publicdomain/zero/1.0/) applies to the data made available in this article, unless otherwise stated. 
Background The fresh fruit provides an ample and reliable source of food for people. The majority of plant hormones-including auxin, cytokinins (CKs), gibberellins (GAs), brassinolide (BR), jasmonic acid (JA), salicylic acid (SA), abscisic acid (ABA), and ethylene-participate in the development and ripening of fresh fruits [1-3]. However, the role of strigolactones (SLs), which were accepted as a new type of plant hormones in 2008 [4, 5], in the development of fresh fruits is unclear.

SLs are a small group of strigol-type compounds. The first natural SL, strigol, was isolated from cotton root exudates as a potent seed germination stimulant of the root-parasitized weed Striga lutea [6]. Other SLs have been identified in diverse plant species [7]. In 2008, SLs were found to have the ability of inhibiting plant branching, so they were accepted as a new type of plant hormones that modulate plant development $[4,5]$. The physiological functions of SLs have been widely excavated in the past decade, and several genes involved in their biosynthetic and signaling pathways have been identified $[8,9]$.

SLs are carotenoid derivatives belonging to terpene lactones, and five major components have been identified in SL biosynthetic pathway: isomerase DWARF27 (D27), Carotenoid Cleavage Dioxygenase 7 (CCD7), Carotenoid Cleavage Dioxygenase 8 (CCD8), cytochrome P450 monooxygenase MORE AXILLARY GROWTH 1 (MAX1), and oxidoreductase-like enzyme LATERAL BRANCHING OXIDOREDUCTASE (LBO) $[9,10]$. First, trans- $\beta$-carotene is catalyzed by D27 to produce 9-cis- $\beta$-carotene, which is cleaved by CCD7 into 9-cis- $\beta$-apo-10'-carotenal. Next, CCD8 converts this cleavage product into carlactone $(\mathrm{CL})$, the biosynthetic precursor for all known type of SLs. The biosynthesis of $\mathrm{CL}$ from trans- $\beta$-carotene is conserved, while the pathway from CL to active SLs varies among plant species. In Arabidopsis, CL is converted by MAX1 to carlactonoic acid (CLA) via 19-hydroxy-carlactone. CLA is subsequently converted into methyl carlactonoate (MeCLA) by an unknown methyl transferase. MeCLA has some activity in shoot branching suppression, a classic SL role, but LBO can further convert MeCLA into an unidentified SL-like compound that may be the final product of SL biosynthesis and have more SL activity in Arabidopsis. In rice, CL is converted into CLA by the MAX1 homolog Os900 (CYP711A2), which also catalyzes the conversion of CLA to 4-deoxyorobanchol (4DO). Subsequently, a second rice MAX1 homolog, Os1400 (CYP711A3), converts 4DO into orobanchol, which has $\mathrm{SL}$ activity. In addition, CL is assumed to be the precursor of 5-deoxystrigol, which can be converted into sorgomol (a sorghum strigol-like SL), although evidence for this is limited [9]. The genes encoding D27, CCD7, CCD8, MAX1, and LBO have been identified in several plant species, such as Arabidopsis D27/rice D27 [11, 12], Arabidopsis MAX3/rice HTD1/pea RMS5/petunia DAD3/tomato CCD7 [13-17], Arabidopsis MAX4/rice D10/pea RMS1/petunia DAD1/tomato CCD8 [18-22], Arabidopsis $M A X 1 /$ rice $M A X 1$ s/petunia $M A X 1$ [23-25], and Arabidopsis LBO [10]. Mutants in these genes display an increased number of tillers or branches and a dwarf phenotype; both can be rescued by SL treatment. However, several components of the SL biosynthesis pathway remain to be identified [9].

One SL signaling pathway has been investigated extensively in Arabidopsis and rice. This pathway has five major components: D14, D3/MAX2, D53/SMXLs, TPR2, and IPA1 $[9,26]$. The SL receptor D14 is an $\alpha / \beta$ hydrolase [27-29], and D3 is an F-box protein that forms a ubiquitination complex with an SCF-type ubiquitin ligase [30, 31]. D53 has been identified as a key transcriptional repressor in SL signaling [32-34]. In the absence of SLs, D14 cannot interact with D3 and D53, while D53 can interact with the transcriptional co-repressor TOPLESS (TPL)-related protein TPR2 and the transcription factor Ideal Plant Architecture1 (IPA1) to repress the transcriptional activity of IPA1. The end result is suppression of the expression of downstream IPA1-regulated genes and no SL response, such as increased tiller or branches. Inversely, In the presence of SLs, D14 binds and hydrolyzes SL molecules, triggering a conformational change in D14 to form a complex with D3 and D53, resulting in D53 degradation via the $\mathrm{SCF}^{\mathrm{D} 3}$ ubiquitination complex, then IPA1 cannot form the complex in the D53- and TPR2-dependent process, resulting in expression of IPA1-regulated genes and an SL response [26, 35].

Prior work on SLs has focused on their roles in Arabidopsis and rice in morphogenesis, root structure, shoot branching, stem elongation, leaf morphology, and stress resistance $[8,9,36]$. However, little is known about the role of SLs in fresh fruit development. Cultivated strawberry is an octaploid non-climacteric fresh fruit, and the complexity of its genome hampers molecular, genetic, and functional studies. The ease of genetic transformation of diploid strawberries, particularly woodland strawberry (Fragaria vesca), has led to their use in the study of rosaceae plants and non-climacteric fresh fruits [37]. In this study, we identified genes involved in SL biosynthesis and signaling, and analyzed their expression patterns in woodland strawberry vegetative organs and fruits at different developmental stages. The results suggested that SLs play a role in the development of woodland strawberry flower and immature fruit. Our findings will facilitate further studies of the relationship between SLs and the development of strawberry fruits. 


\section{Results}

Identification of SL biosynthetic genes D27, MAX1, and $\angle B O$ in woodland strawberry

D27 is a 9-cis/all-trans- $\beta$-carotene isomerase and has been functionally analyzed in rice and Arabidopsis [11, 12]. We used the amino acid sequences of the rice and Arabidopsis D27 proteins as queries to identify similar proteins in rice, Arabidopsis, apple, peach, maize, and woodland strawberry protein databases, and constructed a phylogenetic tree. The identified proteins clustered into clades 1,2 , and 3 . Clades 1 and 2 together formed a larger group with a probability of 99\% (Additional file 1). Rice OsD27 and Arabidopsis AtD27 belonged to clade 1, which we named the D27 family. Interestingly, the clade 1/D27 family included one rice protein (OsD27), one Arabidopsis protein (AtD27), and one peach protein (Prupe.8G233300), but two apple proteins (MDP0000186801 and MDP0000155610) and two maize proteins (GRMZM2G116461 and GRMZM2G158175). Apple MDP0000186801 and MDP0000155610 clustered together with a bootstrap value of 99 , as did maize GRMZM2G116461 and GRMZM2G158175 with a bootstrap value of 100 (Additional file 1; Fig. 1a), suggesting that D27 underwent duplication in apple and maize during evolution. In woodland strawberry, only mrna20277 clustered in clade 1/D27, with a bootstrap value of 99 (Fig. 1a), suggesting that it participates in SL biosynthesis. In addition, a motif analysis using MEME showed that the $\mathrm{N}$-terminal motifs of the proteins differed among plant species, including between rice OsD27 and Arabidopsis AtD27. However, the C-terminus of both rice OsD27 and Arabidopsis AtD27 harbors motifs 1, 2, 4, and 9, which may be important for D27 activity. The D27 proteins in apple, peach, maize, and woodland strawberry also contain these four motifs (Fig. 1a; Additional file 2; Additional file 3). Therefore, we named mrna20277 of woodland strawberry as FveD27.

SL biosynthetic genes FveCCD7 and FveCCD8 of woodland strawberry have been identified in a previous study [38], as the downstream component of CCD7 and CCD8, the $M A X 1$ gene family was identified in Arabidopsis, rice, woodland strawberry, maize, apple, and peach in our study. MAX1, a cytochrome P450 monooxygenase, participates in the biosynthesis of SLs. Cytochrome $\mathrm{P} 450$ proteins can be divided into 10 clans: CYP71, CYP72, CYP85, CYP86, CYP51, CYP74, CYP97, CYP710, CYP711, and CYP727. SL biosynthetic gene MAX1 belongs to the CYP711 clan [39] (Additional file 4). In this study, two woodland strawberry proteins (mrna02706 and mrna02708), three peach proteins (1G410000, 1G410100, and 1G410300), five maize proteins (GRMZM2G070508, GRMZM2G147119, AC2 09853.2_FGP001, GRMZM2G018612, and GRMZM2 G023952), and six apple proteins (MDP0000215198, MDP0000231714, MDP0000130133, MDP0000148030, MDP0000171153, and MDP0000677258) clustered in CYP711 together with Arabidopsis AtMAX1 and petunia PhMAX1, which are related to SL biosynthesis (Fig. 1b). In addition, seven rice proteins (Os02g0221900, Os06g0565100, Os01g0701500, LOC_Os01g50520, Os0 1g0700900, Os01g0701400, and LOC_Os01g50570) clustered in CYP711 (Fig. 1b), but only Os01g0700900 (Os900) and Os01g0701400 (Os1400) are involved in SL biosynthesis [24]. A motif composition analysis showed that most of the proteins (including all of the functional analyzed MAX1 proteins in Arabidopsis, petunia, and rice) in CYP711 contain 9-15 motifs. However, GRMZM2G147119, AC209853.2_FGP001,
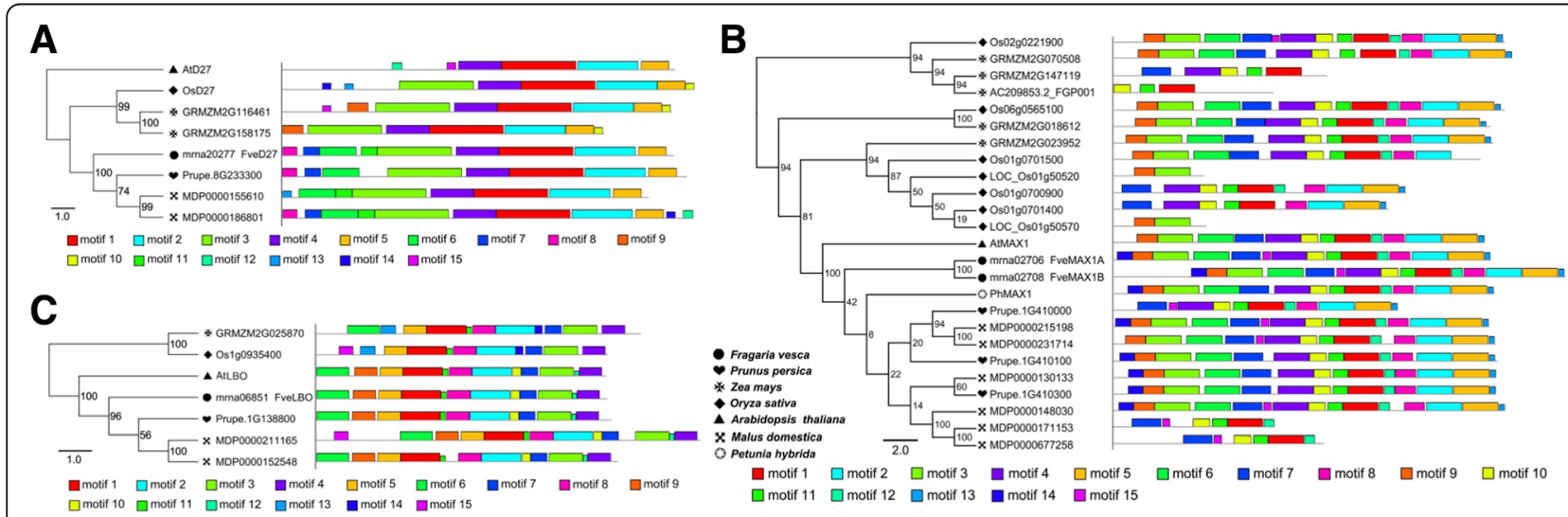

Fig. 1 Conserved motif analysis of D27, MAX1 and LBO protein families from rice, Arabidopsis, apple, peach, maize, petunia, and woodland strawberry. a The motif composition of D27 family. $\mathbf{b}$ The motif composition of MAX1 family. c The motif composition of LBO family. Motifs were identified using MEME software, up to 15 motifs were permitted and other parameters were at the default settings. The 15 motifs were indicated by boxed of different color. Gray lines represent non-conserved sequences. Distribution and protein sequences of conserved motifs were presented in Additional files 2 and 3. rice (Oryza sativa), Arabidopsis (Arabidopsis thaliana), apple (Malus domestica), peach (Prunus persica), maize (Zea mays), woodland strawberry (Fragaria vesca), and petunia (Petunia hybrida) 
LOC_Os01g50520, LOC_Os01g50570, MDP0000171153, and MDP0000677258 contain 2-6 motifs, suggesting that these six proteins do not have MAX1 activity. In woodland strawberry, mrna02706 and mrna02708 have the same motif composition composed by 15 motifs, similar to Arabidopsis AtMAX1 and petunia PhMAX1 (Fig. 1b; Additional file 2; Additional file 3). Therefore, mrna02706 and mrna02708 in woodland strawberry are likely orthologs of MAX1 and participate in SL biosynthesis; we named these proteins FveMAX1A and FveMAX1B, respectively.

$L B O$, which encodes an oxidoreductase-like enzyme, acts in the final stages of SL biosynthesis in Arabidopsis. LBO belongs to the DOXC54 clade of the 2-oxoglutarate and $\mathrm{Fe}(\mathrm{II})$-dependent dioxygenase superfamily [10] (Additional file 5). We used the amino acid sequence of Arabidopsis LBO as a query to identify similar proteins in Arabidopsis, rice, wood strawberry, maize, apple, and peach, and constructed a phylogenetic tree using PhyML. The tree showed that LBO is highly conserved, one protein of Arabidopsis (LBO), rice (Os01g0935400), woodland strawberry (mrna06851), maize (GRMZM 2G025870), and peach (1G138800), and two proteins (MDP0000211165 and MDP0000152548) of apple were included in the DOXC54 clade. MDP0000211165 and MDP0000152548 clustered together with a bootstrap value of 100 (Additional file 5), suggesting gene duplication. Further, woodland strawberry mrna06851, peach 1G138800, and apple MDP0000152548 have identical motif composition composed by 12 motifs as Arabidopsis LBO, while maize GRMZM2G025870, rice Os01g0935400, and apple MDP0000211165 have the following differences at the N-terminal: GRMZM2G025870 has motifs 13 and 14 instead of motifs 9,10 , and 12; Os01g0935400 has motifs 13-15 instead of motifs 6, 9, 10, and 12; and MDP0000211165 has one additional motif (motif 15) (Fig. 1c; Additional file 2; Additional file 3 ). The homologous genes in rice, woodland strawberry, maize, apple, and peach have not been studied, and so we named mrna06851 of woodland strawberry FveLBO.

\section{Identification of the SL receptor D14 in woodland strawberry}

$D 14 / D A D 2$, which encodes an $\alpha / \beta$ hydrolyzyme, is the SL receptor gene in the plant SL signal transduction pathway [27-29]. We used the sequences of the D14 proteins from rice (OsD14), Arabidopsis (AtD14), and petunia (PhDAD2) with known function as queries to identify homologs in rice, Arabidopsis, maize, apple, peach, and woodland strawberry, and constructed a phylogenetic tree (Fig. 2a). The phylogenetic tree was divided into six clades: Clade1-Clade6. Clade1 and 2 clustered in a large group, with a bootstrap value of 77 . The SL receptors OsD14, AtD14, and PhDAD2 were clustered into clade 1, and the KAR receptors OsD14L and AtKAI2 into clade 2, so we named clades 1 and 2 the D14 and D14L families, respectively. D14 family comprised one protein from rice (OsD14), Arabidopsis (AtD14), petunia (PhDAD2), woodland strawberry (mrna02565), and peach (Prupe.1G423400), two proteins from maize (GRMZM2G008751 and GRMZM2G 077127), and three from apple (MDP0000529739, MDP0000888050, and MDP0000898597) (Fig. 2a). Similarly, D14L family comprised one rice protein (OsD14L), one Arabidopsis protein (AtKAI2), one woodland strawberry protein (mrna02565), one peach protein (Prupe.6G225000), two maize proteins (GRMZM2G074138 and GRMZM2G113866), and six apple proteins (MDP0000178428, MDP0000218555, MDP0000274383, MDP0000228645, MDP0000127844, and MDP000013 6111) (Fig. 2a). Therefore, we named mrna02565 and mrna23992 as FveD14 and FveD14L of woodland strawberry, respectively. Interestingly, D14 and D14L proteins of maize and apple clustered together with a high bootstrap value, indicating the occurrence of gene duplications.

To investigate functional conservation during evolution, we analyzed motif composition using MEME. The motif composition was conserved within each clade, but divergent among different clades (Additional file 2; Additional file 3; Additional file 6). D14, D14L, and clade 3 have relatively similar motif composition compared to Clade4, Clade5 and Clade6. The majority of proteins in D14 and D14L have identical motif compositions, while most clade 3 proteins lack motif 3 compared to D14 and D14L families, suggesting involvement of motif 3 in binding SLs and KAR. In addition, apple MDP0000218555, MDP0000274383, and MDP0000228645 in D14L, and MDP0000120479 and MDP0000430391 in clade 3, are truncated proteins with four or five motifs, and so may have functions different from the other D14L and clade 3 proteins (Additional file 2; Additional file 3; Additional file 6). From the result, the identical motif composition of D14 and D14L proteins hampered determination of their functions.

In order to further study the difference of conserved amino acid sequences between D14 and D14L, We next performed a ClustalW multi-sequence alignment of D14 proteins (OsD14, AtD14, PhDAD2, and FveD14) and D14L proteins (AtKAI2, OsD14L, and FveD14L) (Fig. 2b). At the amino acid level, the putative hydrolase catalytic triad residues of FveD14 (Ser98-Asp219-His248) and FveD14L (Ser95-Asp217-His246) were identical to those of AtD14 (Ser97-Asp218-His247), OsD14 (Ser 147-Asp268-His297), PhDAD2 (Ser96-Asp217-His246), AtKAI2 (Ser95-Asp217-His246), and OsD14L (Ser96-Asp218-His247). This suggests that the putative SL receptor FveD14 and the KAR receptor FveD14L from woodland strawberry have hydrolase activity. Seven 
A

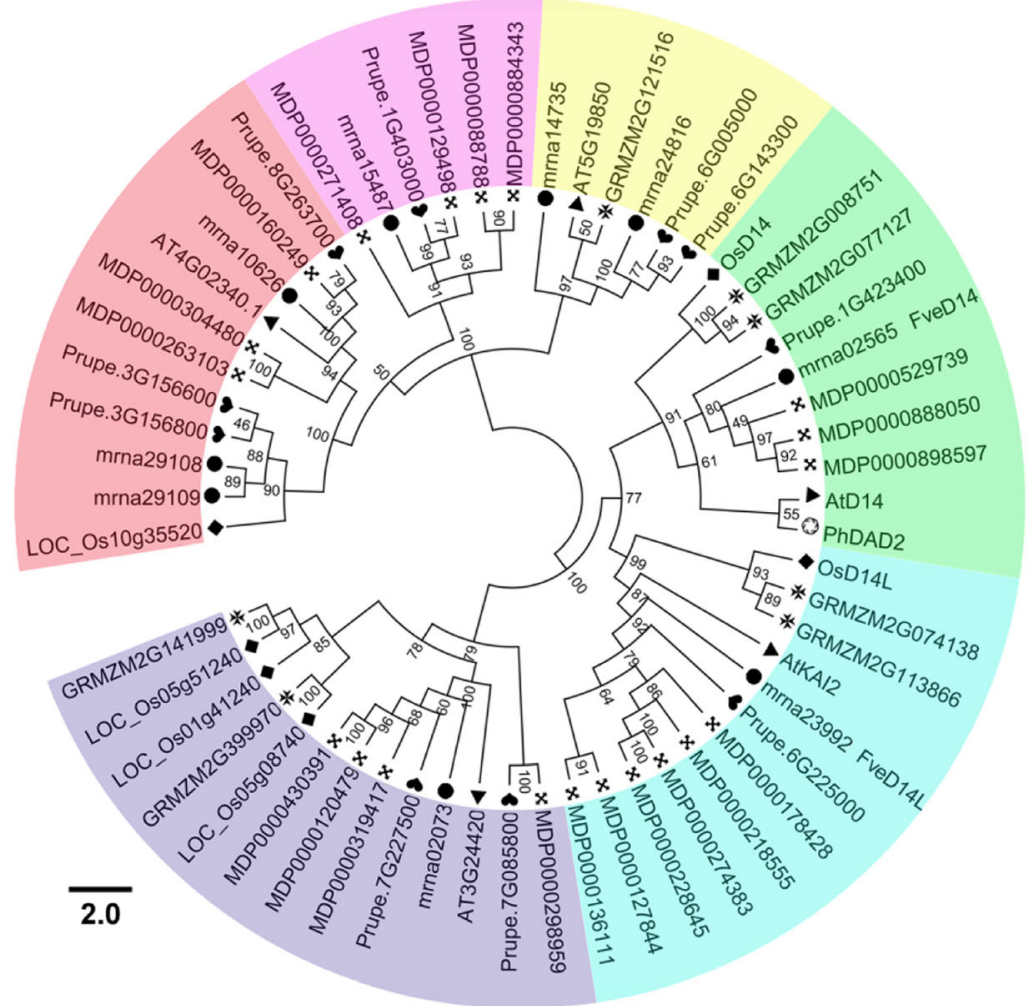

Clade1/D14

Clade2/D14L

Clade3

Clade4

Clade5

Clade6

Fragaria vesca
Prunus persica
Arabidopsis thaliana
Oryza sativa
Zea mays
Malus domestica
Petunia hybrida

B

FveD14

AtD14

PhDAD2

OSD14 MLRSTHPPPSSPSSSSGGGGGGGSSASSSSEKTMVGGGGGGGGGSGSAAP

FveD14L

AtKAl2

OsD14L

FveD14

ATD14 DQSAWQR I LPYFKPNYR I IVYDL

PhDAD2 DQSAWNRI LPFFLRDYRVVLYDL

OsD14 DQSAWSRVLPYLTRDHRVVLYDLVCAGSVNPDHFDFRRYDNLD

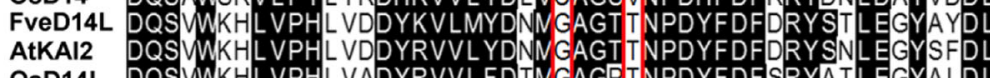

OsD14L DQSWWKHLVPHL VADYRVVLFDTNGAGPTNPDYFDFSRYATLEGYALDLL

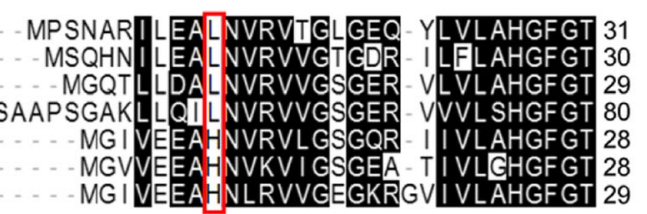
AtD14 ELFSKLIL IGFSPRFLNDEDYHGGFEEGE I EKVFSAMEANYEAWVHGF APLAVGADV PAAVREF SRTLFNMRPD I SLFVS 191 PhDAD2 ELFSKLILIGASPRFLNDEDYHGGFEQGE I EKVFSAMEANYEAWVNGFAPLAVGADV PAAVREF SRTLFNMRPD ITLFVS 190 OsD14 DLFAKLVL IGASPRFLNDSDYHGGFELEE IQQVFDAMGANYSAWATGYAPLAVGADV PAAVQEF SRT LFNMRPD I SLHVC 241 FveD14L DLFTKL VMI AASPR YLNDSDYYGGFEQEDLEQLFEA IRSNYKAWC SGFAPLAVGGDMDSVAVQEF SRT LFNMRPDI ALSVA 190 AtKAI2 DLFSK I VMI SASPRY VNDVDYOGGFEQED LNQLFEA IRSNYKAWCLGFAPLAVGGDMDS I AVQEF SRTLFNMRPD I ALSVG 190 OSD14L DLFSKLVLLSASPRYLNDVDYYGGFEQEDLDELFEAMGSNYKAWCSGFAPLCVGGDMESVAVQEF SRTLFN IRPD I ALSVA 191

FveD14 RAVFNSDLREFLGFVRVPCFIIQTARDFISVPASVANYLKDHLGGRNTIVTLETEGHLPHLSAPGLLARKLRCCLLSS . . . 269 AtD14 RTVFNSDLRGVLGLVRVPTCVIQTAKDVSVPASVAEYLRSHLGGDTTVETLKTEGHLPQLSAPAQLAQFLRRALPR .... 267 PhDAD2 RTVFNSDMRGVLGLVKVPCH IFQTARDHSVPASVATYLKNHLGGKNTVHWLN IEGHLPHL SAPTLLAQELRRALSHR - . 267 OSD14 QTVFKTDLRGVLGVRAPCVVVQTTRDVSVPASVAAYLKAHLGGRTTVEFLQTEGHLPHLSAPSLLAQVLRRALARY ... 318 FveD14L QT I FQSDMRQV I GMVTVPCHI LQSVKDLAVPVVVTEYLHQNLGGESIVEVMSSDGHLPOLSSPDIVIPVLLRH IRHDIAV 270 ATKAI2 QT I FQSDMRQI LPFVTVPCHILQSVKDLAVPVVVSEYLHANLGCESVVEVI PSDGHLPQLSSPDSVIPVILRHIRNDIAM 270 OSD14L QT IFQSDVRSLLPLVTVPCHIVQSTKDLAVPVVVSEYLHKHLGGDSIVEVMPSEGHLPQLSSPD IVIPVLLRHIQHDIAV 271

Fig. 2 Maximum likelihood phylogeny and sequence alignment of D14 family. a Phylogenetic tree of D14 family identified from rice (Oryza sativa), Arabidopsis (Arabidopsis thaliana), apple (Malus domestica), peach (Prunus persica), maize (Zea mays), petunia (Petunia hybrida), and woodland strawberry (Fragaria vesca). b Sequence alignment of predicted D14 and D14L proteins of woodland strawberry (Fve) with the functionally analyzed D14 and D14L proteins of rice (Os), Arabidopsis (At), and petunia (Ph). The putative hydrolase catalytic triad residues of D14 and D14L were highlighted in blue boxes, and the seven specificity-determining positions (SDPs) which determine the functions of D14 and D14L proteins were highlighted in red boxes 
specificity-determining positions (SDPs) determine the functions of D14- and D14L-related proteins. In our results, the seven SDPs of FveD14 (L11-C56-V60-F127-P170-S221-H251) were identical to those of other known functional D14 proteins (rice OsD14, Arabidopsis AtD14, and petunia PhDAD2), and SDPs of FvD14L (H8-G53-T57-Y124-S168-A219-Q249) were identical to those of other known D14L proteins (rice OsD14L and Arabidopsis AtKAI2) (Fig. 2b). These findings confirm that FveD14 and FveD14L are putative SL and KAR receptors, respectively, in woodland strawberry.

\section{Identification of $D 3$ and $D 53 \mathrm{SL}$ signaling genes in woodland strawberry}

D3/MAX2 gene encodes an F-box leucine-rich repeat (LRR) protein that participates in SL signaling [30, 31]. The F-box protein family is a superfamily, and D3 proteins belong to the F-box LRR_7 family [40]. D3 genes have been identified in rice (OsD3), Arabidopsis (AtMAX2), pea (PsRMS4), and petunia (PhMAX2A, PhMAX2B) [25, 30, 41, 42]. According to the phylogenetic tree, one woodland strawberry protein (mrna15755), one peach protein (3G117700), three apple proteins (MDP0000137221, MDP0000305017, and MDP0000466825), and two maize proteins (GRMZM2G393272 and GRMZM2G405203) clustered with OsD3, AtMAX2, PsRMS4, PhMAX2A, and PhMAX2B (Additional file 7). We next analyzed the motif composition of four F-box LRR_7 protein families according to Arabidopsis (bootstrap value > 90) (Fig. 3a; Additional file 2; Additional file 3). These four clusters we named the MAX2/D3, AFB, VFB, and EBF clades. The motif composition was conserved within each clade, but widely divergent among the clades. In the MAX2/ D3 clade, the D3 proteins of dicotyledons (Arabidopsis,

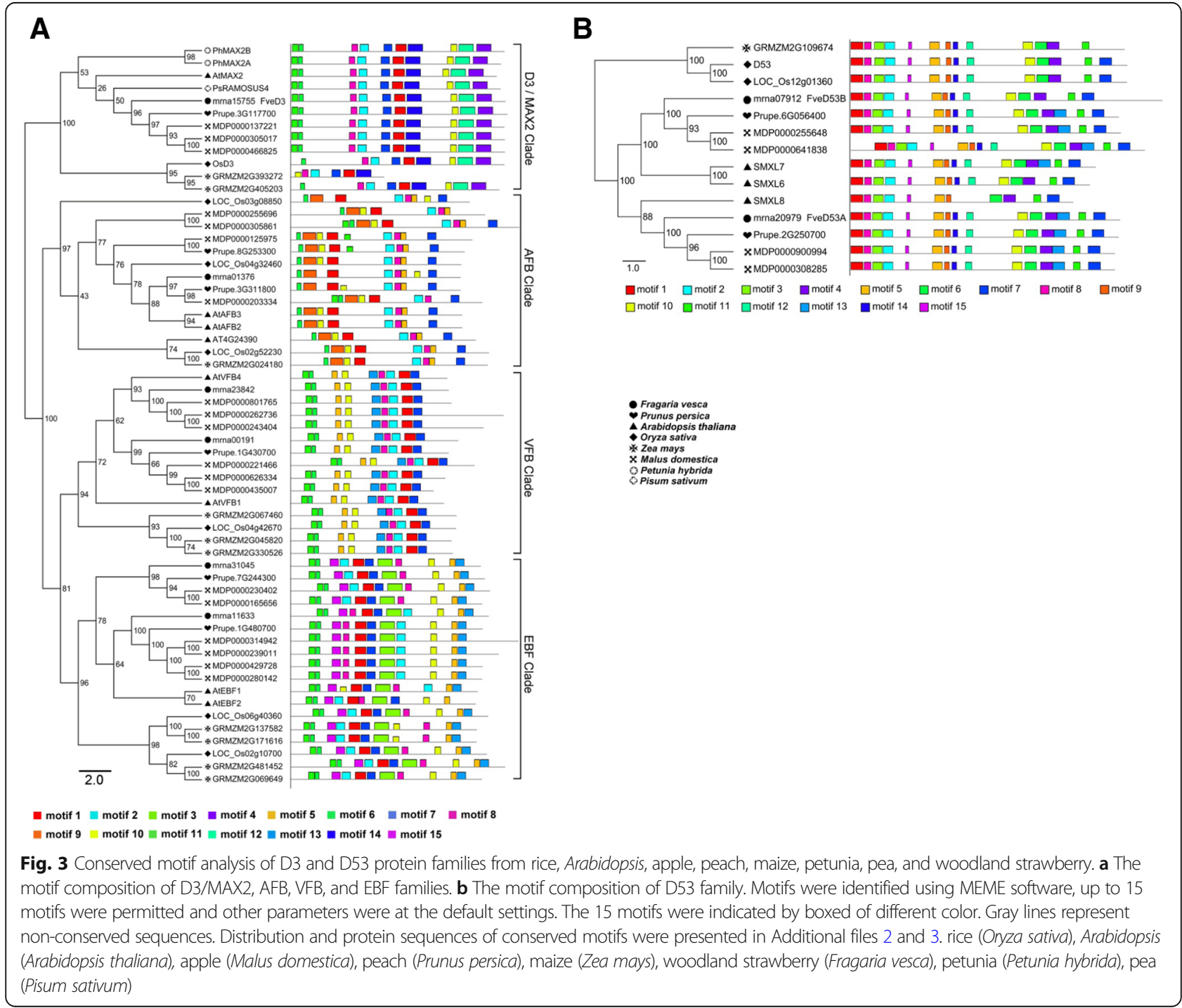


pea, petunia, woodland strawberry, peach, and apple) were clustered together, and these proteins contain 10 motifs, while the D3 proteins of monocotyledons (rice and maize) which clustered together only contain nine motifs, with the exception of GRMZM2G393272. GRMZM2G393272 is a truncated protein that lacked three C-terminal motifs, and thus may have a different function. The motif structure of woodland strawberry mrna15755 is consistent with that of MAX2/D3 clade proteins (Fig. 3a; Additional file 2; Additional file 3), suggesting a role in SL signaling; we thus named mrna15755 as FveD3.

D53/SMXL6/SMXL7/SMXL8 acts as a repressor of SL signalling and its degradation is induced by SLs [32-34]. Using rice D53 as a query, we identified 84 proteins in rice, Arabidopsis, apple, peach, maize, and woodland strawberry after removing redundant proteins. The resulting phylogenetic tree showed that the 84 proteins clustered into the D53-like, SMAX1-like, and ClassIClp ATPase clades (Additional file 8). D53-like and SMAX1-like clustered together to form a larger group, with a bootstrap value of 97 . The SL signaling proteins D53/SMXL6/SMXL7/SMXL8 belong to the D53-like family, which also contains two rice proteins (D53 and Os12g01360), three Arabidopsis proteins (SMXL6, SMXL7, and SMXL8), two woodland strawberry proteins (mrna20979 and mrna07912), one maize protein (GRMZM2G109674), two peach proteins (Prupe.6G056400 and Prupe.2G250700), and four apple proteins (MDP0000255648, MDP0000641838, MDP000090 0994, and MDP0000308285) (Additional file 8). Arabidopsis SMXL7 contained 15 motifs, while SMXL6, SMXL8, and rice D53 lacked one, four, and one motif, respectively, but these four proteins all have been demonstrated as SL signaling proteins. Maize GRMZM2G109674 lacked motif 7 compared to rice D53; but all D53 homologs of peach and apple were consistent with Arabidopsis SMXL7. Interestingly, woodland strawberry mrna20979 has 15 motifs, consistent with Arabidopsis SMXL7, while mrna07912 has 14 motifs, consistent with Arabidopsis SMXL6 (Fig. 3b; Additional file 2; Additional file 3). Thus, the function of mrna20979 and mrna07912 is likely to be identical to that of D53, and so we named mrna20979 as FveD53A and mrna07912 as FveD53B.

\section{Expression of SL biosynthetic and signaling genes in woodland strawberry vegetative tissues}

To assess the role of SLs in vegetative tissue growth and development of woodland strawberry, the expression levels of SL biosynthetic and signaling genes in the root, stem, leaf, and petiole of woodland strawberry were analyzed by qPCR. The result showed that, in strawberry seedlings, the expression of $F v e D 27$ was low in root and slightly higher in stem and petiole, but FveD27 expression in leaf was several hundred-fold higher than that in stem and petiole (Fig. 4a). FveCCD7 was expressed mainly in root and stem, and at a low level in leaf; indeed, $F v e C C D 7$ expression in stem was approximately nine-fold that in leaf, and four-fold higher in petiole than in leaf (Fig. 4b). The expression of FveCCD8 was highest in stem, at approximately 150 -fold that in root, but was not expressed in leaf or petiole (Fig. 4c). In addition, the expression of FvMAX1A and FvMAX1B was highest in stem, lowest in root, and moderate in leaf and petiole (Fig. 4d, e). FveLBO was highly expressed in stem, at approximately 2.7 -fold that in leaf, and low expressed in root and petiole (Fig. 4f). For SL signaling genes, the transcript level of the SL receptor-encoding gene FveD14 was highest in leaf, being threefold that in root, stem, and petiole (Fig. 4g), and the expression of FveD3 in leaf was six-fold that in root, stem, and petiole (Fig. 4h). In addition, FveD53A and FveD53B had the highest expression in leaf, moderate in stem, and the lowest expression in root and petiole (Fig. 4i, j). To summarize, the putative SL biosynthetic genes in woodland strawberry showed the highest expression in stem, except for $F v D 27$, while the putative SL signaling genes showed the highest expression levels in leaf. Therefore, SL biosynthesis and signal transduction activity may differ among the tissues of woodland strawberry plants.

\section{Expression of SL biosynthetic and signaling genes in woodland strawberry flower and early fruits}

To investigate the role of SL in woodland strawberry reproductive development, expression data of SL biosynthetic and signaling genes were obtained from Strawberry Genomic Resources (http://bioinformatics.towson.edu/strawberry/) [43] (Additional file 9), and are presented as heat maps. Strawberry flower undergoes 13 developmental stages, which are divided into three parts: stages 1-4, early flower development; stages 5-7, initiation and development of reproductive organs; and stages 8-13, differentiation of floral organs [44]. The expression levels of the putative SL biosynthetic and signaling genes differed among both tissue types and developmental stages (Fig. 5). The transcript levels of the SL biosynthetic genes FveD27, FveCCD7, FveCCD8, FveMAX1A, FveMAX1B, and FveLBO were generally higher in young complete flowers (1-4 flowered_B_C, 5-6 perianth, and 6-7 receptacle) compared to other mature flower tissues (7-12 carpels and anthers), but the transcript levels of the SL signaling genes FveD14, FveD3, and FveD53A were low (Fig. 5a). During carpel development, the transcript levels of FveMAX1A, Fve$M A X 1 B$, and FveLBO initially decreased and subsequently increased. The transcript levels of $F v D 27$, 


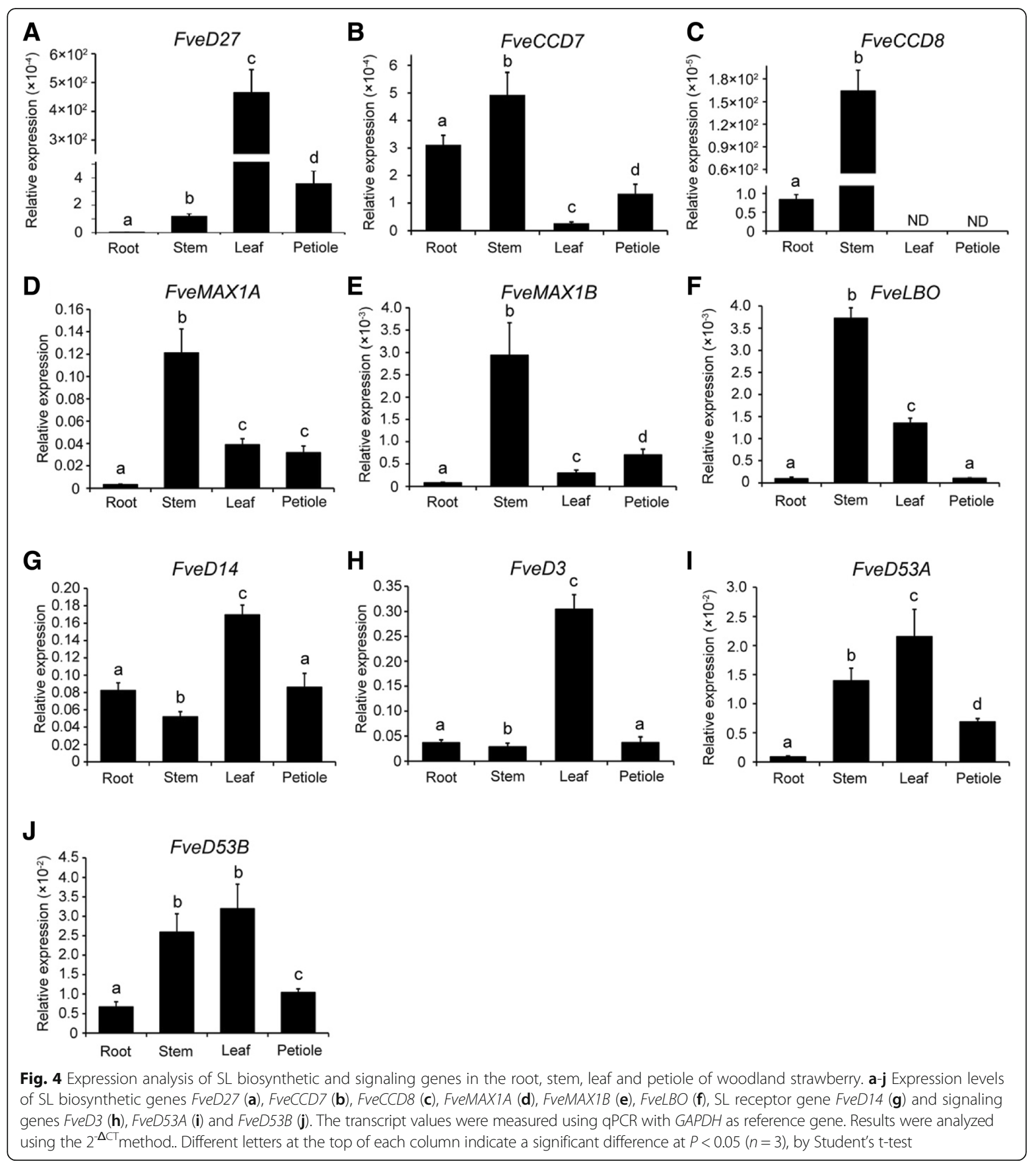

FveCCD7, and FveCCD8 were very low or undetectable. By contrast, the transcript levels of the putative SL signaling genes FveD14, FveD3, FveD53A, and FveD53B were high in the developing carpel (Fig. 5a). During anther development, the transcript levels of SL biosynthetic genes were similar to those in the developing carpel, while those of the SL signaling genes were lower than in the developing carpel. In addition, the transcript levels of SL biosynthetic and signaling genes in anther decreased from stages 9-12, suggesting reduced SL activity (Fig. 5a). Interestingly, stage 10 microspores exhibited high and low transcript levels of SL biosynthetic and signaling genes, respectively, whereas the transcript levels of the biosynthetic and signaling genes were low 


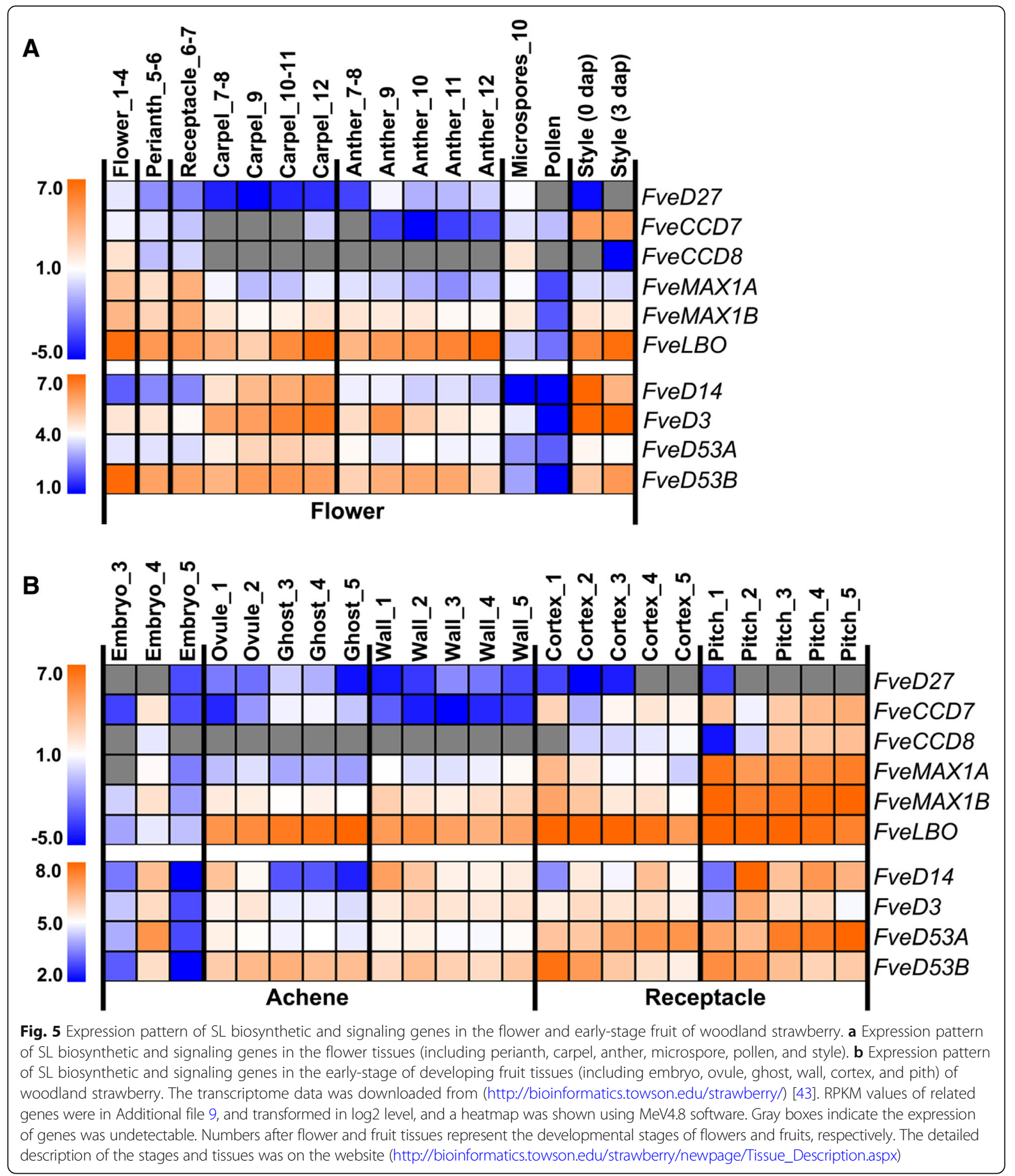

in mature pollen. In addition, the transcript levels of FveCCD7, FveAMX1A, FveMAX1B, and FveLBO were high in style before and after pollination, but those of FveD27 and FveCCD 8 were negligible. Notably, the transcript levels of FveD14 and FveD3 were high in style
(Fig. 5a), suggesting active SL signaling before and after pollination.

Early fruit development of woodland strawberry from anthesis to green fruit is divided into five stages: stage 1 (pre-fertilization), stage 2 (2-4 days post-anthesis 
[DPA]), stage 3 (6-9 DPA), stage 4 (8-10 DPA), and stage 5 (10-13 DPA) [43]. The ovule and ovary wall at stages 1-2 showed high transcript levels of FveMAX1A, FveMAX1B, FveLBO, FveD14, FveD3, and FveD53s, while those of the SL biosynthetic genes FveD27, FveCCD7, and FveCCD8 were low or undetectable. Achene at stages 3-5 was divided into embryo, ghost (seeds without embryos), and wall. The transcript levels of $F v M A X 1 A$ and $F v M A X 1 B$ in wall increased slightly from stages 3-5, while those of FveLBO, FveD14, FveD3, and FveD53s showed little or no change, and the transcript levels of FveD27, FveCCD7, and FveCCD8 were low or negligible. In developing ghosts, the transcript levels of most biosynthetic and signaling genes were low, while those of FveD27, FveCCD7, and FveLBO were high in stage 3-4 ghosts (Fig. 5b). During embryonic development of early achene, the expression patterns of SL biosynthetic and signaling genes show distinct characteristics. Expression of all of the SL biosynthetic and signaling genes was low at embryo stage 3 , but increased at embryo stage 4 (with the exception of $F v D 27$ ) and decreased at embryo stage 5 (Fig. $5 \mathrm{~b}$ ), suggesting that SLs are important for embryonic development at stage 4 . The receptacle was divided into the cortex and pith. The FveD27 transcript level was low or undetectable in developing cortex and pith, but those of other SL biosynthetic genes were higher. In addition, the transcript levels of SL signaling genes were high in cortex and pith at stages 2-5. Notably, the transcript levels of FveD14 and FveD3 increased significantly after pollination in cortex and pitch (stages 1-2) (Fig. 5b), suggesting activation of SL signaling in receptacle due to pollination.

\section{Expression of SL biosynthetic and signaling genes during woodland strawberry fresh fruit development}

To investigate the role of SLs in the development and ripening of woodland strawberry fruits, the expression of SL biosynthetic and signaling genes was analyzed by qPCR in receptacles lacking seeds at the small green (SG), large green (LG), small white (SW), large white $(\mathrm{LW})$, pink $(\mathrm{P})$, and red $(\mathrm{R})$ stages $[45,46]$. The result showed that the overall expression of FveD27 in the receptacle was low, consistent with the transcriptome data. The expression of FveD27 was highest in SG receptacle, approximately four-fold that in LG and SW receptacles. At the LW, P, and R stages, FveD27 transcript was barely detected (Fig. 6a). The expression of FveCCD7 increased from the SG stage, peaked at the LG stage, decreased significantly at the $\mathrm{SW}$ stage, and was very low at the $\mathrm{LW}, \mathrm{P}$, and $\mathrm{R}$ stages (Fig. 6b). FveCCD8, FvMAX1A, $F v M A X 1 B$, and $F v e L B O$ had similar expression profiles; their expression was highest at the SG stage, and gradually decreased to a low level at the LW, P, and R stages (Fig. 6c-f). Expression of the SL signaling genes FvD14,
$F v D 3, F v e D 53 A$, and $F v e D 53 B$ was highest at the SG and LG stages, and decreased thereafter. Interestingly, unlike the SL biosynthetic genes, expression of the SL signaling genes was high at the ripening stages (LW, P, and R) (Fig. $6 \mathrm{~g}-\mathrm{j}$ ). Indeed, the expression of FveD14 and FveD3 increased at the $\mathrm{P}$ stage, and decreased at the $\mathrm{R}$ stage (Fig. 6g, h). Further, we measured the concentration of strigol (one native SL) in woodland strawberry receptacle at five developmental stages (SG, LG, SW, P and R stages) by HPLC-ESI-MSn. The result showed that the concentration of strigol was also high at early stages (SG and LG), and then decreased from SW stage to ripening stages ( $\mathrm{P}$ and $\mathrm{R}$ ) (Fig. 6k). To summarize, the expression of SL biosynthetic and signaling genes is high in early developing fruits but decreases as fruit development and ripening proceed, consistent with SL concentrations, suggesting that SLs play a role in the early development of strawberry fruits.

\section{Discussion \\ Identification of SL biosynthestic and signaling genes in woodland strawberry}

SLs play important roles in plant growth and development, and their biosynthetic and signaling genes in Arabidopsis and rice have been investigated extensively. Arabidopsis and rice have one each of the D27, CCD7, $C C D 8, D 14$, and $D 3$ genes, which participate in SL biosynthesis and signaling $[8,9]$. In woodland strawberry, there is also only one protein clustered together with Arabidopsis and rice D27, CCD7, CCD8, D14, and D3, respectively, and these gene families have similar motifs and amino acid sequences (Fig. 1-3);. D27, CCD7, and CCD8 are essential for SL biosynthesis, and D14 and D3 for SL recognition and signal transduction $[8,9]$; therefore, $F v e D 27, F v e C C D 7$, and $F v e C C D 8$ likely participate in SL biosynthesis, and FveD14 and FveD3 in SL signaling. Arabidopsis has one MAX1 gene (At2g26170), which participates in the production of SL precursors [23], whereas rice has five (Os01g0700900, Os01g0701400, Os01g0701500, Os02g0221900, and Os06g0565100) [24]. Interestingly, Os01g0701500, Os02g0221900, and Os06g0565100 are not involved in the biosynthesis of SL precursors despite having motifs similar to those of Arabidopsis MAX1. By contrast, Os01g0700900 and Os01g0701400, which lack four motifs present in Arabidopsis MAX1 (Fig. 1b), catalyze the biosynthesis of active SLs [24]. The two FveMAX1 proteins (FveMAX1A and FveMAX1B) of woodland strawberry have the same motif composition as Arabidopsis MAX1, but the $\mathrm{N}$-terminal amino acid sequence of FveMAX1B is of unknown function (Fig. 1b). Thus, FveMAX1A, similar to Arabidopsis MAX1, likely catalyzes the production of SL precursors, while the function of FvMAX1B is unclear. Further studies should evaluate the functions of 


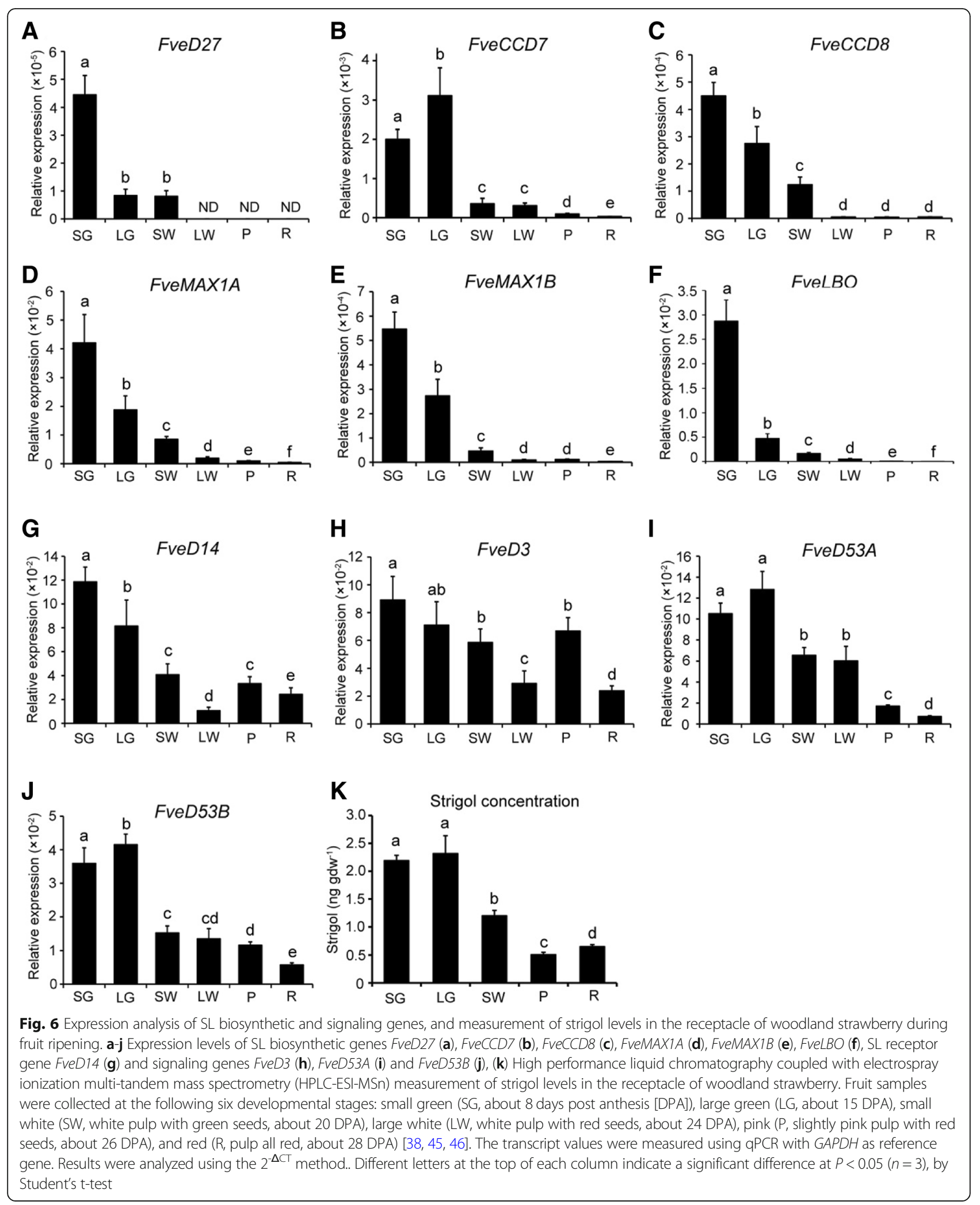


FveMAX1A and FveMAX1B in SL biosynthesis. In addition, Arabidopsis, rice, and woodland strawberry have one $L B O$ gene with the same motif composition (Fig. 1c); Arabidopsis LBO acts in the final stages of SL biosynthesis to produce active SLs [10]. Based on our speculation that FveMAX1A has similar activity to Arabidopsis MAX1, we postulate that FveLBO has a similar function to Arabidopsis LBO in the final stages of SL biosynthesis. Two rice proteins (OsD53 and LOC_Os 12g01360), three Arabidopsis proteins (AtSMXL6, AtSMXL7, and AtSMXL8) and two woodland strawberry proteins (mrna07912 and mrna20979) clustered in the D53 family of SL signaling proteins (Fig. 3b). OsD53, AtSMXL6, AtSMXL7, and AtSMXL8 can interact with D14 and form complexes with D3/MAX2, resulting in ubiquitination and degradation of OsD53, AtSMXL6, AtSMXL7, and AtSMXL8 in an SL-responsive manner [32-34]. However, the function of rice LOC_Os12g0136, which has high homology to OsD53, is unknown [32]. Woodland strawberry mrna07912 clustered with AtSMXL6 and AtSMXL7, while mrna20979 clustered with AtSMXL8. These proteins have similar motif compositions (Fig. 3b), so whether mrna07912 and mrna2097 have similar functions as the other D53 proteins is unclear.

\section{Expression patterns of SL biosynthetic and signaling genes in vegetative organs of woodland strawberry}

SLs are synthesized in plant roots, and can be transported to shoots via the transpiration stream, as suggested by the wild-type (WT) branching pattern of SL biosynthetic mutant scions grafted onto WT rootstocks $[11,47]$. In addition, plant shoots may also produce SLs, based on the WT branching phenotypes generated by grafting of WT scions onto rootstocks of Arabidopsis and pea SL biosynthetic mutants [15, 23, 48]. In Arabidopsis, the expression of the SL biosynthetic genes $M A X 3 / C C D 7$ and $M A X 4 / C C D 8$ was lower and higher, respectively, in root compared with shoot. By contrast, the MAX1 transcript levels were similar in root and shoot. Notably, the expression of $D 27$ was extremely low in root [11]. In rice, the expression of $D 27, C C D 7 /$ $H T D 1$, and $C C D 8 / D 10$ is lower in root than in stem [12, 14, 19]. However, this does not mean that localized high concentrations of SLs in certain cell types in the root do not exit, especially some biosynthetic genes, such as D27, CCD7/HTD1, CCD8/D10, MAX1, and MAX4/ $C C D 8$ predominantly expressed in root vascular tissue $[12,14,19,23,49]$. In fact, natural SLs have been isolated from the roots of diverse plant species [7], while low concentrations of SLs were detected in sorghum shoots [50]. Therefore, we speculate that SLs are produced in root and shoot of woodland strawberry because of the expression of SL biosynthetic genes in root and shoot (Fig. 4a-f), but the expression pattern provides no guidance on where the SL concentrations are highest. D27, CCD7/HTD1 and CCD8/D10, are also expressed in rice leaf $[12,14,19]$, as are $C C D 7$ and $C C D 8$ in tomato leaf $[17,22]$. In our study, expression of $D 27$ and $C C D 7$, but not $C C D 8$, was detected in leaf and petiole of woodland strawberry (Fig. 4a-c), possibly because CCD8 is not expressed in these tissues or is expressed at a level below the limit of detection. If the former is true, no CL is produced because D27, MAX3, and MAX4 act on non-mobile substrates in the plastid [11, 47]. However, FveMAX1A, FveMAX1B and FveLBO, which act downstream of $C C D 8$, were expressed in leaf and petiole (Fig. $4 \mathrm{~d}-\mathrm{f})$, so that SLs may be produced in these locations using CL transported from other tissues.

AtD14/OsD14 is the only SL receptor investigated to date [27-29], and MAX2/D3 is essential for ubiquitin-mediated protein degradation [30, 31]; thus, AtD14/OsD14 and MAX2/D3 are essential for the roles of SLs in the development of different tissues. Consistent with the roles of SLs in plant root and shoot branching, the expression of $A t D 14 / O s D 14$ and $M A X 2 / D 3$ is similar in the shoot/stem and root of Arabidopsis and rice [28, $30,41,51]$. In woodland strawberry, the expression of FveD14 and FveD3 is similar in root and stem (Fig. 4g, h). D53 represses SL signaling in rice tillers and Arabidopsis branching, and its expression is relatively high in the shoot base of rice seedlings and axillary buds $[32,33]$, as well as in the stem of woodland strawberry (Fig. I, J). Because the runners and crowns of strawberry differentiate from stem axillary buds [52], the high expression levels of FveD53A and FveD53B suggest that these two genes play roles in the development of runners and crowns. In addition, the expression of FveD14, FveD3, and FveD53s was high in leaf and moderate in petiole of woodland strawberry (Fig. 4g-j). The high expression of these genes in leaf is also seen in Arabidopsis, pea, petunia, and rice $[15,20,28,30,32,33,41,51]$. In Arabidopsis, SLs promote the expansion of leaf blade and petiole, producing more open rosettes [9], and regulate leaf senescence in concert with ethylene [53]. In Medicago, SLs increase leaf serration but the overall leaf shape was not affected [54]. In addition, there is a cross-talk between SL and ABA in integrating stress signals to regulate leaf stomatal development and function [36]. However, The effects of SLs on leaf development are complex and deserves in-depth thought and research.

\section{Possible role for $\mathrm{SL}$ in the reproductive growth of woodland strawberry}

Floral development is important in plant reproductive growth and is regulated by the plant hormones auxin, GAs, CKs, ethylene, and ABA [55]. For example, auxin 
not only determines the initiation of flower primordia but also specifies the number of floral organs and pattern formation within a floral organ [56, 57]; CKs positively regulate ovule formation and pistil development [58], and CK response factors integrate the auxin and CK pathways for female reproductive organ development [59]; and GAs act positively in the termination of vegetative development but inhibits flower formation [60, 61]. Ethylene senses DNA damage in the female anther primordium and promotes female flower formation in $\mathrm{cu}-$ cumber [62]. Expression of the SL biosynthetic and signaling genes in rice is high in panicle and even higher than root $[12,14,19,32,33,41,51]$, suggesting that SLs participate in panicle development, but no study focus on this so far. In our study, the transcript levels of putative SL biosynthetic and signaling genes were high and low, respectively, in woodland strawberry flower (stages 1-4), perianth (stages 5-6), and receptacle (stages 6-7) (Fig. 5a), suggesting SLs may accumulate, but SL signaling was inactive in these tissues at the early stages. We speculate that SL accumulation plays a role in progression to the next developmental stage, particularly the carpel (stages 7-8) from the receptacle base (stages 67), because FveD14 expression is activated in carpel at stages 7-8 (Fig. 5a). During carpel and anther development, expression of the SL biosynthetic genes FveD27, FveCCD7, and FveCCD8 was low or undetectable (Fig. $5 \mathrm{a})$, indicating that $\mathrm{CL}$ is not produced in the carpel and anther. However, the high expression of FveMAX1s and FveLBO in these tissues suggests synthesis of SLs. The expression of SL signaling genes was higher in carpel than in anther, and the expression of FveD14 increased with carpel development (Fig. 5a), suggesting SLs play a role in carpel and pistil development. In addition, the expression of biosynthetic and signaling genes was all extremely low in mature pollen, we speculate that SLs are not active in mature pollen. However, expression of FveD14 was high in the styles (including stigmas) of just-opened flowers but decreased after pollination (Fig. 5a), so fertilization may be required for the functions of SLs in the female organs of woodland strawberry.

\section{SLs may play a role in the early development of woodland strawberry fruits}

Fruit set, development, and ripening are associated with the activities of various plant hormones. Fruit set is defined as the activation of a developmental program that converts the ovary into a developing fruit. Auxin, GAs, and CKs are involved in fruit set $[2,3,63]$. After tomato fertilization, the levels of auxin and CKs dramatically increase to a peak at 2 DPA, and auxin activates GA biosynthesis [64]. In this study, the expression of SL biosynthetic genes decreased upon fertilization both in the achene and receptacle (Fig. 5b), suggesting a decrease in the SL levels in these tissues during fertilization. However, expression of the SL receptor gene FveD14 decreased in the ovule and carpel/ovary wall of achene, but increased in the receptacle cortex and pitch, upon fertilization (Fig. 5b). Thus, SL signaling may be activated in receptacle, but inhibited in achene by fertilization. This suggests that SLs play different roles in achene and receptacle during fertilization.

In tomato, auxin, CKs, and GAs cooperate to regulate the early fruit development. Auxin, CKs and GAs all have high levels in this process. A summary of the idea was proposed that auxin, CKs and GAs impact each other to promote cell division and expansion, and determine fruit size [65-67]. ABA, BR, and ethylene also play roles in the early development of fruits $[2,3,64]$. In strawberry, the levels of indole-3-acetic acid (IAA), $\mathrm{GA}_{1}$, and castasterone increase early in fruit development and decrease prior to color accumulation [68]. The expression of auxin and GA biosynthetic genes is high in the achene (embryo, ghost, and wall) and low in the receptacle (pith and cortex), suggesting that auxin and GA biosynthesis occurs mainly in the achene [43, 69]. However, auxin receptor genes are expressed in both the achene and receptacle, and the expression of GA receptor genes is higher in the receptacle than in the achene. Therefore, auxin signaling is active in the achene and receptacle, and GA signaling in the receptacle. Based on this, the following model has been proposed: auxin is synthesized and stimulates GA biosynthesis in the seed, and auxin and GAs are transported to the receptacle to promote receptacle growth [43]. Consistent with this, removal of achenes inhibits receptacle growth, and removal of some achenes results in abnormally shaped fruits because only the parts of the receptacle adjacent to the remaining achenes continue to grow [70]. Exogenous application of $\mathrm{GA}_{3}$, 1-naphthaleneacetic acid (NAA), or $\mathrm{GA}_{3}$ plus NAA to non-pollinated fruits induces receptacle enlargement [43]. Therefore, auxin and GAs produced in seeds play important roles in fruit development, but the roles of other hormones in strawberry fruit development are unclear. In our study, the expression of SL biosynthetic and signaling genes was higher in receptacle, particularly in developing pitch, than in achene (Fig. 5b), so SLs may play important roles in receptacle development. However, unlike auxin and GAs, SLs may be synthesized and accumulate in the receptacle to promote its development. Interestingly, in achene, the expression of SL biosynthetic and signaling genes was high in the walls of developing carpel and ovary, ovule (stages 1-2), and embryo (stage 4), but low in ghost (stages 3-5) and embryo (stages 3 and 5) (Fig. $5 \mathrm{~b})$. Thus, the roles of SLs differ among tissues and developmental stages. 
The ripening of fleshy fruits is an important developmental process in which plant hormones play important roles. Ethylene is the major regulator of most aspects of fruit ripening in climacteric fruits, such as tomato, although auxin, ABA, and GAs are also involved [1-3]. In non-climacteric fruits, such as strawberry, ABA is the major regulator of fruit ripening $[68,71]$. Besides, auxin and brassinosteroid also have high levels, and the expression of some biosynthetic and signaling genes increase at the late stages of receptacle ripening $[69,72]$. However, exogenous applications of NAA delayed receptacle ripening [73], while exogenous application of BR promote ripening [72]. Ethylene is considered not to be related to strawberry ripening, but influences seed maturation and metabolism [73, 74]. The expression of some JA signaling-related genes decreases from the fruit development to ripening stages, suggesting JA plays a role in early strawberry fruit development, which also correlates negatively with the beginning of the ripening process [75]. SlCCD7 expression was highest in tomato fruits at the immature green stage [17]. Similarly, in woodland strawberry, expression of the SL biosynthetic and signaling genes was high in the early stages of receptacle development and decreased significantly in ripening receptacle, which was consistent with the change of SL concentrations (Fig. 6). Therefore, we speculate that SLs participate in the regulation of early development, but not ripening, of woodland strawberry fruits. However, understanding of the regulatory mechanisms of SLs in fruit development is still limited. SLs can interact with auxin, CKs and GAs in regulating root development, shoot branching and seed germination [76], together with auxin, CKs, GAs and SLs all have high levels in the early developing fruit, and auxin, CKs and GAs can cooperate to regulate the early fruit development. Therefore, we speculate that SLs regulate the early fruit development through interacting with other hormones, such as auxin, CKs and GAs.

\section{Conclusions}

In conclusion, we identified one $D 27$, two $M A X 1$, and one $L B O$ gene in the SL biosynthetic pathway, and one D14, one D3, and two D53 genes in the SL signaling pathway in woodland strawberry. The expression levels of the SL biosynthetic and signaling genes differed among plant tissues and developmental stages. The expression of the SL biosynthetic and signaling genes was high in early receptacle and decreased during receptacle development, suggesting that SLs play a role in woodland strawberry fruit development, especially at the early stages. Woodland strawberry is a useful model plant for studying the molecular and developmental biology of non-climacteric fruits, and our findings provide insight into the function of SLs in fruit development.

\section{Methods}

Identification of genes and phylogenetic analyses

Protein sequences and annotation information of two monocots (Oryza sativa and Zea mays) and four eudicots (Fragaria vesca, Arabidopsis thaliana, Prunus persica, and Malus domestica) were downloaded from Phytozom (https://phytozome.jgi.doe.gov/pz/portal.html) (Additional file 10). SL-related genes were identified based on review articles (Additional file 11) $[8,9]$. The longest sequence was selected while there was redundancy because of alternative splicing. Protein domains were validated using SMART (http://smart.embl-heidelberg.de/), Pfam (http://pfam.xfam.org/search\#tabview=tab1), and CDD (https://www.ncbi.nlm.nih.gov/Structure/cdd/wrpsb.cgi).

Phylogenetic trees were visualized in FigTree v. 1.3.1 software.

The D27 protein family has no domain annotation on the InterPro website and is classified in the 'domain of unknown function' family (Pfam: DUF4033) [11]. To identify D27 homologs in woodland strawberry, a BLASTp search $\left(E-v a l u e ~<10^{-4}\right)$ was performed in the protein databases of six species using the OsD27 and AtD27 sequences as queries in BioEdit. The sequences were filtered to remove redundancy, and the presence of a DUF4033 domain was verified using SMART, Pfam, and CDD. Finally, the sequences of 21 proteins (including OsD27 and AtD27) were obtained. The proteins were aligned in ClustalX 2.1 and exported as FASTA and PHYLIP files. The best JTT + I + G model was identified by Model-Generator [77]. Subsequently, PHYLIP files were used to construct maximum likelihood phylogenetic trees in PhyML with 100 replicates [78].

Using the AtMAX1, PhMAX1, Os01g0700900, Os01g0701400, Os01g0701500, Os02g0221900, and Os06g0565100 sequences as queries, we performed a local BLASTp (E-value $<10^{-25}$; other parameters were at their default values) search of the protein databases of six species in BioEdit. Redundant sequences and those that lacked a P450 domain (Pfam: P450) were removed by validation using SMART, Pfam, and CDD, yielding approximately 200 protein sequences. The proteins were aligned in ClustalX 2.1 (using the default parameters) and exported as FASTA and PHYLIP files. The best JTT + G + F model was identified by Model-Generator. The PHYLIP files were used to generate maximum likelihood phylogenetic trees in PhyML with 100 replicates.

To identify LBO homologs in Oryza sativa, Arabidopsis thaliana, Zea mays, Fragaria vesca, Prunus persica and Malus domestica, the amino acid sequences of Arabidopsis LBO protein was used as query to performed a local BLASTp (E-value $<10^{-50}$ ) search in BioEdit. After removing redundancy, 2OG-FeII_Oxy domain were identified using the SMART, Pfam and CDD databases. Subsequently, all the obtained amino acid sequences 
were multiply aligned by ClustalX 2.1(using default parameters), and the optimum model JTT + I + G was determined by Model-Generator. Finally, a phylogenetic tree was constructed using PhyML with 100 replicates.

Using three OsD14, AtD14, and PhDAD2 as queries, a local BLASTp (E-value $<10^{-4}$ ) search for homologs in the protein databases of six species was performed in BioEdit. After removing redundancy, the remaining sequences were submitted to SMART, Pfam, and CDD to verify the presence of an $\alpha / \beta$ hydrolyzyme domain. Next, FASTA and PHYLIP files of the 58 identified proteins were obtained by alignment in ClustalX 2.1 with the default parameters. The best WAG $+\mathrm{I}+\mathrm{G}+\mathrm{F}$ model was identified by Model-Generator. A maximum likelihood tree was constructed in PhyML using the default settings and 100 bootstrap replicates.

To identify D3 homologs, we performed a local BLASTp (E-value $<10^{-4}$ ) search in BioEdit software using OsD3, AtMAX2, PsRMS4 and PhMAX2A, PhMAX2B as the query. After removing redundancy, LRR domains were identified using the SMART, Pfam, and CDD databases. In total, 153 F-box LRR proteins (including OsD3, AtMAX2, PsRMS4, PhMAX2A, and PhMAX2B) were aligned using ClustalX 2.1 with the default parameters, and the optimum JTT + G + F model was determined by Model-Generator. A phylogenetic tree was constructed using PhyML. The phylogenetic tree was reconstructed based on four selected groups (bootstrap value > 90) of F-box LRR_7 related proteins [40].

We identified D53 homologs by Local BLASTp $\left(\right.$ E-Value $\left.<10^{-4}\right)$ in BioEdit using rice D53 protein as query. After removing redundancy which did not belong to the Clp (Caseinolytic peptidase) proteins validated by SMART, Pfam and CDD, 84 proteins were multiply aligned by ClustalX 2.1 (using default parameters) for FASTA and PHYLIP format files. The optimum JTT + G + F model was identified by Model-Generator. Finally, PHYLIP format file was used to construct a phylogenetic tree by PhyML with 100 replicates.

\section{Conserved motif and multiple sequence alignment analysis}

Conserved motifs were identified by submitting the complete amino acid sequences to the MEME website (Version 4.10.2, http://meme-suite.org/- tools/meme) [79]; up to 15 motifs were permitted and other parameters were at the default settings. Multiple sequence alignments were performed using ClustalW with the default parameters in BioEdit software. Seven SDPs were decided [28], and putative hydrolase catalytic triad residues were identified [11].

\section{Plant materials}

Seeds of Ruegen (Fragaria vesca) were from a seven-generation inbred line provided by Janet Slovin.
Woodland strawberry seedlings were grown in $10 \times 10$ $\mathrm{cm}$ pots in a climate room, under a $16 \mathrm{~h}$ light $\left(22^{\circ} \mathrm{C}\right) / 8 \mathrm{~h}$ dark $\left(20^{\circ} \mathrm{C}\right)$ cycle with $65 \%$ relative humidity. The flowering time was tagged, and fruit samples were collected at the following six developmental stages: SG (about 8 days DPA), LG (about 15 DPA), SW (white pulp with green seeds, about 20 DPA), LW (white pulp with red seeds, about 24 DPA), P (slightly pink pulp with red seeds, about $26 \mathrm{DPA}$ ), and R (pulp all red, about 28 DPA) $[38,45,46]$. After removing seeds, the receptacle samples were stored at $-80^{\circ} \mathrm{C}$.

Ruegen seeds were sterilized in $75 \%$ alcohol for $5 \mathrm{~min}$ followed by $2 \%(w / v)$ sodium hypochlorite for $7 \mathrm{~min}$ and washed several times in sterile water. The aseptic seedlings were grown in a climate chamber under a $16 \mathrm{~h}$ light $\left(22^{\circ} \mathrm{C}\right) / 8 \mathrm{~h}$ dark $\left(20^{\circ} \mathrm{C}\right)$ cycle with $55 \mu \mathrm{mol} \mathrm{m}{ }^{-2} \mathrm{~s}^{-1}$ irradiance provided by white fluorescent lamps. Murashige and Skoog medium $(2 \%[\mathrm{w} / \mathrm{v}]$ sucrose, $7.2 \mathrm{~g} / \mathrm{L}$ agar) was used for growth of the seedlings. Following growth for up to 40 days, the root (entire root tissue), stem (dwarf stem, entire tissue after removing the petiole and hypocotyl), leaf (all leaves except cotyledons), and petiole were collected. Three biological replicates were performed, each comprising eight seedlings.

\section{RNA extraction and qPCR analysis}

The root, stem, leaf, petiole and receptacle samples stored at $-80{ }^{\circ} \mathrm{C}$ were used for total RNA extraction with a modified CTAB method [80].The cDNA was generated by Primerscript RT reagent Kit with gDNA Erase (Takara) according to the manufacturer's protocol. Quantitative PCR (qPCR) was performed using SYBR Premix Ex Tag (Takara), with GAPDH as an internal reference gene [81]. Results were analyzed using the $2^{-\triangle C T}$ method [81]. Three biological and three technical replicates were performed and analyzed. Primers used in this study are listed in Additional file 12.

\section{Expression analysis}

The transcriptome data for different development stages of woodland strawberry were downloaded from (http:// bioinformatics.towson.edu/strawberry/) [43]. RPKM values of related genes were transformed in $\log 2$ level, and a heatmap was shown using MeV4.8 software.

\section{SL analysis}

The measurement of strigol levels was carried out by high performance liquid chromatography coupled with electrospray ionization multi-tandem mass spectrometry (HPLC-ESI-MSn) at Beijing Forestry University [82-84]. ${ }^{2} \mathrm{H}_{3}$-GR24 was synthesized according to Guo et al. (2015) [85]. 


\section{Plant materials and purification procedure}

Extraction and prepurification of endogenous SLs from strawberry receptacles were performed according to Liu et al. (2013) [82] with some modifications. Strawberry receptacles were ground to powders using liquid nitrogen, and dehydrated by freeze-drying before hormone analyses. 100 mg dry weight sample was extracted overnight at $4{ }^{\circ} \mathrm{C}$ in 6 $\mathrm{ml} 80 \%$ methanol containing $100 \mathrm{ng}{ }^{2} \mathrm{H}_{3}$-GR24 as the internal standard. After centrifugation, the supernatant was collected and the residue was re-extracted for $60 \mathrm{~min}$ as above. The combined supernatant was loaded onto the Sep-Pak Plus C18 cartridge, and washed with $2 \mathrm{~mL} \mathrm{5 \%}$ methanol, and eluted with $2 \mathrm{~mL} 80 \%$ methanol. The elution was evaporated to aqueous phase in a vacuum at $4{ }^{\circ} \mathrm{C}$, and then loaded onto the MAX cartridge. After being washed with $1 \mathrm{M}$ FA, $0.1 \mathrm{M} \mathrm{NH} 4 \mathrm{OH}$, and $0.1 \mathrm{MNH} 4 \mathrm{OH}$ in $60 \% \mathrm{MeOH}$, the MAX cartridge was eluted with $1.25 \mathrm{M}$ FA in $70 \% \mathrm{MeOH}$. The obtained elution was dried under vacuum and redissolved in $50 \mu \mathrm{L}$ of $50 \%$ methanol in $0.001 \%$ formic acid. After filtering, $10 \mu \mathrm{L}$ of the solution was injected into the HPLC-ESI-MSn system for strigol analysis according to the following optimum procedure. Each sample was analyzed in triplicate.

\section{Analysis procedure of strigol}

Before analyzing strigol levels of plant samples, the standard sample of strigol purchased from OLChemim Ltd. (Olomouc, Czech Republic) was used to optimize parameters to obtain maximal signal intensities of strigol in HPLC-ESI-MSn. The optimum procedure was as follows: The elution gradient of HPLC was performed with 95\% A (0.1\% formic acid in water) and 5\% B (methanol) at constant flow rate of $0.15 \mathrm{~mL} / \mathrm{min}$ for $1 \mathrm{~min}$; A decreased to $60 \%$ and B increased to $40 \%$ in 9 min; then $\mathrm{A}$ decreased to 0 and $\mathrm{B}$ increased to $100 \%$ at $20 \mathrm{~min}$, which should hold on for $5 \mathrm{~min}$; after that, $\mathrm{A}$ and $\mathrm{B}$ reached the original percent in $1 \mathrm{~min}$ and held on for $5 \mathrm{~min}$. Then, a LCQ DECA XP MAX ion trap mass spectrometer system (Thermo-Finnigan) coupled with an ESI source was used with the following parameters: positive ionization mode, Capillary temperature $300^{\circ} \mathrm{C}$, sheath gas: nitrogen, 30abi units, Aux gas: nitrogen, 5abi units (ca. $3.33 \mathrm{~L} / \mathrm{min}$ ), Capillary voltage $+4.0 \mathrm{kV}$, Tube Lens Offset $30 \mathrm{~V}$, Multiple RF Amplifier 400Vp-p, Multiple 1 Offset $-6.80 \mathrm{~V}$, Multiple 2 Offset $-9.50 \mathrm{~V}$, Inter-multiple Lens Voltage - $16.00 \mathrm{~V}$, Entrance Lens $-50 \mathrm{~V}$, Trap DC Offset Voltage $-10 \mathrm{~V}$, Capillary temperature $280^{\circ} \mathrm{C}$, Collision energy $35 \mathrm{eV}$.

\section{Additional files}

Additional file 1: Phylogenetic analysis of D27 protein family identified from rice, Arabidopsis, apple, peach, maize, and woodland strawberry. The amino acid sequences of D27 from rice and Arabidopsis were used as queries. 21 proteins were obtained after removing redundancy, and the Phylogenetic tree was construct in PhyML with 100 replicates. (JPG $214 \mathrm{~kb})$

Additional file 2: Distribution of conserved motifs in different protein families. Motif analysis was performed online by MEME, (Version 4.10.2, http://meme-suite.org/- tools/meme) [79]; up to 15 motifs were permitted and other parameters were at the default settings. (DOCX 36 $\mathrm{kb})$

Additional file 3: Protein sequences of conserved motifs in different protein families. (DOCX $22 \mathrm{~kb}$ )

Additional file 4: Phylogenetic analysis of CYP450 protein family identified from rice, Arabidopsis, apple, peach, maize, and woodland strawberry. AtMAX1, PhMAX1, Os01g0700900, Os01g0701400,

Os01g0701500, Os02g0221900, and Os06g0565100 sequences were used as queries, approximately 200 protein sequences were obtained after removing redundancy, and the Phylogenetic tree was construct in PhyML with 100 replicates. (JPG $1427 \mathrm{~kb}$ )

Additional file 5: Phylogenetic analysis of LBO protein family identified from rice, Arabidopsis, apple, peach, maize, and woodland strawberry. The amino acid sequences of $L B O$ from Arabidopsis were used as queries, approximately 200 protein sequences were obtained after removing redundancy, and the Phylogenetic tree was construct in PhyML with 100 replicates. (JPG $1751 \mathrm{~kb}$ )

Additional file 6: Conserved motif analysis of D14 protein families from rice, Arabidopsis, apple, peach, maize, petunia, and woodland strawberry. Motifs were identified using MEME software, up to 15 motifs were permitted and other parameters were at the default settings. The 15 motifs were indicated by boxed of different color. Gray lines represent non-conserved sequences. Distribution and protein sequences of conserved motifs were presented in Additional files 2 and 3. (JPG $879 \mathrm{~kb}$ )

Additional file 7: Phylogenetic analysis of F-box LRR protein family identified from rice, Arabidopsis, apple, peach, maize, and woodland strawberry. The amino acid sequences of OsD3, AtMAX2, PsRMS4 and PhMAX2A, PhMAX2B were used as queries, $153 \mathrm{~F}$-box LRR protein sequences were obtained after removing redundancy, and the Phylogenetic tree was construct in PhyML with 100 replicates. (JPG $1504 \mathrm{~kb}$ )

Additional file 8: Phylogenetic analysis of D53 protein family identified from rice, Arabidopsis, apple, peach, maize, and woodland strawberry. The amino acid sequences of D53 from rice were used as queries, 84 proteins were obtained after removing redundancy, and the Phylogenetic tree was construct in PhyML with 100 replicates. (JPG $1211 \mathrm{~kb}$ )

Additional file 9: The RPKM value of SL biosynthetic and signaling genes in the flower and early-stage fruit of woodland strawberry. The transcriptome data was downloaded from (http://bioinformatics.towson.edu/strawberry/) [43]. (DOCX $21 \mathrm{~kb}$ )

Additional file 10: Databases for all six species we used in this research. (DOCX $12 \mathrm{~kb}$ )

Additional file 11: The functionally analyzed proteins of plant species used in this study. (DOCX $13 \mathrm{~kb}$ )

Additional file 12: Primers used in $\mathrm{QPCR}$ analysis of this study. (DOCX $13 \mathrm{~kb})$

\section{Abbreviations}

ABA: Abscisic acid; BR: Brassinolide; CCD7: Carotenoid Cleavage Dioxygenase 7; CCD8: Carotenoid Cleavage Dioxygenase 8; CKs: Cytokinins; CL: Carlactone; CLA: Carlactonoic acid; D27: DWARF27; GAs: Gibberellins; IAA: Indole-3-acetic acid; IPA1: Ideal Plant Architecture1; JA: Jasmonic acid; LBO: Oxidoreductaselike enzyme LATERAL BRANCHING OXIDOREDUCTASE; MAX1: Cytochrome P450 monooxygenase MORE AXILLARY GROWTH 1; MeCLA: Methyl carlactonoate; NAA: 1-naphthaleneacetic acid; SA: Salicylic acid; SLs: Strigolactones; TPR2: TOPLESS (TPL)-related protein 2 


\section{Funding}

This work was supported by the National Natural Science Foundation of China (NSFC 31601736), the General Financial Grant Program from the China Postdoctoral Science Foundation (2015 M581812), Special Financial Grant Program from the China Postdoctoral Science Foundation (2016 T90471), the Priority Academic Program Development of Jiangsu Higher Education Institutions (PAPD). The funding bodies did not play any role in the design of the study and collection, analysis, and interpretation of data and in writing the manuscript.

\section{Availability of data and materials}

The datasets supporting the conclusions of this article are included within the article and its Additional files. Protein sequences and annotation information of two monocots (Oryza sativa and Zea mays) and four eudicots (Fragaria vesca, Arabidopsis thaliana, Prunus persica, and Malus domestica) were downloaded from Phytozom (https://phytozome.jgi.doe.gov/pz/portal.html) (Additional file 10). The transcriptome data for different development stages of woodland strawberry were downloaded from (http://bioinformatics.towson.edu/ strawberry/).

\section{Authors' contributions}

HW and YL conceived and designed the project; $H L$ and HW performed most of the experiments; QQ and XJ synthesized the internal standard ${ }^{2} \mathrm{H}_{3^{-}}$ GR24, measured the concentration of strigol by HPLC-ESI-MSn, and analyzed the data of strigol concentration; QD and JX contributed to the RNA extraction and $\mathrm{PPCR}$ analysis; $\mathrm{HL}, \mathrm{HW}, \mathrm{HC}$ and $\mathrm{XH}$ carried out the analysis; $\mathrm{HL}$ and HW wrote the manuscript; JD supervised the bioinformatics analysis of protein families; JD and $Y L$ revised the manuscript. All authors have read and approved the final version of this manuscript.

\section{Ethics approval and consent to participate}

Not applicable.

\section{Consent for publication}

Not applicable.

\section{Competing interests}

The authors declare that they have no competing interests.

\section{Publisher's Note}

Springer Nature remains neutral with regard to jurisdictional claims in published maps and institutional affiliations.

\section{Author details}

${ }^{1}$ State Key Laboratory of Crop Genetics and Germplasm Enhancement, College of Horticulture, Nanjing Agricultural University, Nanjing 210095, China. ${ }^{2}$ Jiangsu Key Laboratory for the Research and Utilization of Plant Resources, Institute of Botany, Jiangsu Province and Chinese Academy of Sciences, Nanjing 210014, China. ${ }^{3}$ National Engineering Laboratory for Tree Breeding, College of Life Sciences and Biotechnology, Beijing Forestry University, Beijing 100083, China. ${ }^{4}$ Department of Plant Science and Landscape Architecture, University of Connecticut, Storrs, CT 06269, USA. ${ }^{5}$ Present address: Fuyang Academy of Agricultural Sciences, Fuyang 236065, China.

\section{Received: 17 October 2018 Accepted: 7 February 2019}

\section{Published online: 14 February 2019}

\section{References}

1. Srivastava A, Handa AK. Hormonal regulation of tomato fruit development: a molecular perspective. J Plant Growth Regul. 2005;24(2):67-82.

2. McAtee P, Karim S, Schaffer R, David K. A dynamic interplay between phytohormones is required for fruit development, maturation, and ripening. Front Plant Sci. 2013:4:79.

3. Kumar R, Khurana A, Sharma AK. Role of plant hormones and their interplay in development and ripening of fleshy fruits. J Exp Bot. 2014;65(16):4561-75.

4. Gomez-Roldan V, Fermas S, Brewer PB, Puech-Pagès V, Dun EA, Pillot JP, Letisse F, Matusova R, Danoun S, Portais JC, et al. Strigolactone inhibition of shoot branching. Nature. 2008;455(7210):189-94.

5. Umehara M, Hanada A, Yoshida S, Akiyama K, Arite T, Takeda-Kamiya N, Magome H, Kamiya Y, Shirasu K, Yoneyama K, et al. Inhibition of shoot branching by new terpenoid plant hormones. Nature. 2008;455(7210): 195-200.

6. Cook CE, Whichard LP, Turner B, Wall ME, Egley GH. Germination of witchweed (Striga lutea Lour.): isolation and properties of a potent stimulant. Science. 1966;154(3753):1189-90.

7. Yoneyama K, Kisugi T, Xie X, Yoneyama K. Chemistry of strigolactones: Why and how do plants produce so many strigolactones? In: de Bruijn FJ, editor. Molecular microbial ecology of the rhizosphere: two volume set. Hoboken, NJ: USA: John Wiley \& Sons; 2013. p. 373-9.

8. Al-Babili S, Bouwmeester HJ. Strigolactones, a novel carotenoid-derived plant hormone. Annu Rev Plant Biol. 2015;66:161-86.

9. Waters MT, Gutjahr C, Bennett T, Nelson DC. Strigolactone signaling and evolution. Annu Rev Plant Biol. 2017:68:291-322.

10. Brewer PB, Yoneyama K, Filardo F, Meyers E, Scaffidi A, Frickey T, Akiyama K, Seto $Y$, Dun EA, Cremer JE, et al. LATERAL BRANCHING OXIDOREDUCTASE acts in the final stages of strigolactone biosynthesis in Arabidopsis. Proc Natl Acad Sci U S A. 2016:113(22):6301-6.

11. Waters MT, Brewer PB, Bussell JD, Smith SM, Beveridge CA. The Arabidopsis ortholog of rice DWARF27 acts upstream of MAX1 in the control of plant development by strigolactones. Plant Physiol. 2012;159(3):1073-85.

12. Lin $H$, Wang $R$, Qian $Q$, Yan M, Meng X, Fu Z, Yan C, Jiang B, Su Z, Li J, et al. DWARF27, an iron-containing protein required for the biosynthesis of strigolactones, regulates rice tiller bud outgrowth. Plant Cell. 2009;21(5): 1512-25.

13. Booker J, Auldridge M, Wills S, McCarty D, Klee H, Leyser O. MAX3/CCD7 is a carotenoid cleavage dioxygenase required for the synthesis of a novel plant signaling molecule. Curr Biol. 2004;14(14):1232-8.

14. Zou J, Zhang S, Zhang W, Li G, Chen Z, Zhai W, Zhao X, Pan X, Xie Q, Zhu L. The rice HIGH-TILLERING DWARF1 encoding an ortholog of Arabidopsis MAX3 is required for negative regulation of the outgrowth of axillary buds. Plant J. 2006;48(5):687-98.

15. Morris SE, Turnbull CG, Murfet IC, Beveridge CA. Mutational analysis of branching in pea. Evidence that Rms 1 and Rms5 regulate the same novel signal. Plant Physiol. 2001;126(3):1205-13.

16. Drummond RS, Martínez-Sánchez NM, Janssen BJ, Templeton KR, Simons JL, Quinn BD, Karunairetnam S, Snowden KC. 2009. Petunia hybrida CAROTENOID CLEAVAGE DIOXYGENASE7 is involved in the production of negative and positive branching signals in petunia. Plant Physiol. 2009; 151(4):1867-77

17. Vogel JT, Walter MH, Giavalisco P, Lytovchenko A, Kohlen W, Charnikhova T, Simkin AJ, Goulet C, Strack D, Bouwmeester HJ, et al. SICCD7 controls strigolactone biosynthesis, shoot branching and mycorrhiza-induced apocarotenoid formation in tomato. Plant J. 2010;61(2):300-11.

18. Sorefan K, Booker J, Haurogné K, Goussot M, Bainbridge K, Foo E, Chatfield S, Ward S, Beveridge C, Rameau C, et al. MAX4 and RMS1 are orthologous dioxygenase-like genes that regulate shoot branching in Arabidopsis and pea. Genes Dev. 2003;17(12):1469-74.

19. Arite T, Iwata H, Ohshima K, Maekawa M, Nakajima M, Kojima M, Sakakibara H, Kyozuka J. DWARF10, an RMS1/MAX4/DAD1 ortholog, controls lateral bud outgrowth in rice. Plant J. 2007;51(6):1019-29.

20. Simons UL, Napoli CA, Janssen BJ, Plummer KM, Snowden KC. Analysis of the DECREASED APICAL DOMINANCE genes of petunia in the control of axillary branching. Plant Physiol. 2007;143(2):697-706.

21. Snowden KC, Simkin AJ, Janssen BJ, Templeton KR, Loucas HM, Simons JL, Karunairetnam S, Gleave AP, Clark DG, Klee HJ. The Decreased apical dominance1/Petunia hybrida CAROTENOID CLEAVAGE DIOXYGENASE8 gene affects branch production and plays a role in leaf senescence, root growth, and flower development. Plant Cell. 2005;17(3):746-59.

22. Kohlen W, Charnikhova T, Lammers M, Pollina T, Tóth P, Haider I, Pozo MJ, de Maagd RA, Ruyter-Spira C, Bouwmeester HJ, et al. The tomato CAROTENOID CLEAVAGE DIOXYGENASE8 (SICCD8) regulates rhizosphere signaling, plant architecture and affects reproductive development through strigolactone biosynthesis. New Phytol. 2012;196(2):535-47.

23. Booker J, Sieberer T, Wright W, Williamson L, Willett B, Stirnberg P, Turnbull C, Srinivasan M, Goddard P, Leyser O. MAX1 encodes a cytochrome P450 family member that acts downstream of $M A X 3 / 4$ to produce a carotenoid derived branchinhibiting hormone. Dev Cell. 2005;8(3):443-9.

24. Zhang $Y$, van Dijk AD, Scaffidi A, Flematti GR, Hofmann M, Charnikhova T, Verstappen F, Hepworth J, van der Krol S, Leyser O, et al. Rice cytochrome P450 MAX1 homologs catalyze distinct steps in strigolactone biosynthesis. Nat Chem Biol. 2014;10:1028-33. 
25. Drummond RS, Sheehan $H$, Simons JL, Martínez-Sánchez NM, Turner RM, Putterill J, Snowden KC. The expression of petunia strigolactone pathway genes is altered as part of the endogenous developmental program. Front Plant Sci. 2012;2:115.

26. Yao R, Li J, Xie D. Recent advances in molecular basis for strigolactone action. Sci China Life Sci. 2018;61(3):277-84.

27. Gaiji N, Cardinale F, Prandi C, Bonfante P, Ranghino G. The computationalbased structure of Dwarf14 provides evidence for its role as potential strigolactone receptor in plants. BMC Res Notes. 2012;5:307.

28. Chevalier F, Nieminen K, Sánchez-Ferrero JC, Rodríguez ML, Chagoyen M, Hardtke CS, Cubas P. Strigolactone promotes degradation of DWARF14, an $\alpha / \beta$ hydrolase essential for strigolactone signaling in Arabidopsis. Plant Cell. 2014;26(3):1134-50.

29. Yao R, Ming Z, Yan L, Li S, Wang F, Ma S, Yu C, Yang M, Chen L, Chen L, et al. DWARF14 is a non-canonical hormone receptor for strigolactone. Nature. 2016:536(7617):469-73.

30. Stirnberg P, Furner IJ, Ottoline Leyser HM. MAX2 participates in an SCF complex which acts locally at the node to suppress shoot branching. Plant J. 2007;50(1):80-94

31. Zhao LH, Zhou XE, Yi W, Wu Z, Liu Y, Kang Y, Hou L, de Waal PW, Li S, Jiang $Y$, et al. Destabilization of strigolactone receptor DWARF14 by binding of ligand and E3-ligase signaling effector DWARF3. Cell Res. 2015;25(11):1219-36.

32. Zhou F, Lin Q, Zhu L, Ren Y, Zhou K, Shabek N, Wu F, Mao H, Dong W, Gan $L$, et al. D14-SCFD3-dependent degradation of D53 regulates strigolactone signalling. Nature. 2013;504(7480):406-10.

33. Jiang L, Liu X, Xiong G, Liu H, Chen F, Wang L, Meng X, Liu G, Yu H, Yuan Y, et al. DWARF 53 acts as a repressor of strigolactone signalling in rice. Nature. 2013;504(7480):401-5.

34. Wang L, Wang B, Jiang L, Liu X, Li X, Lu Z, Meng X, Wang Y, Smith SM, Li J. Strigolactone signaling in Arabidopsis regulates shoot development by targeting D53-like SMXL repressor proteins for ubiquitination and degradation. Plant Cell. 2015;27(11):3128-42.

35. Song X, Lu Z, Yu H, Shao G, Xiong J, Meng X, Jing Y, Liu G, Xiong G, Duan J, et al. IPA1 functions as a downstream transcription factor repressed by D53 in strigolactone signaling in rice. Cell Res. 2017;27(9):1128-41.

36. Ha CV, Leyva-González MA, Osakabe Y, Tran UT, Nishiyama R, Watanabe Y, Tanaka M, Seki M, Yamaguchi S, Dong NV, et al. Positive regulatory role of strigolactone in plant responses to drought and salt stress. Proc Natl Acad Sci U S A. 2014;111(2):851-6.

37. Shulaev V, Sargent DJ, Crowhurst RN, Mockler TC, Folkerts O, Delcher AL, Jaiswal P, Mockaitis K, Liston A, Mane SP, et al. The genome of woodland strawberry. Nat Genet. 2011;43(2):109-16.

38. Wang Y, Ding G, Gu T, Ding J, Li Y. Bioinformatic and expression analyses on carotenoid dioxygenase genes in fruit development and abiotic stress responses in Fragaria vesca. Mol Gen Genomics. 2017;292(4):895-907.

39. Nelson DR, Schuler MA, Paquette SM, Werck-Reichhart D, Bak S. Comparative genomics of rice and Arabidopsis. Analysis of 727 cytochrome P450 genes and pseudogenes from a monocot and a dicot. Plant Physiol. 2004;135(2):756-72.

40. Xu G, Ma H, Nei M, Kong H. Evolution of F-box genes in plants: different modes of sequence divergence and their relationships with functional diversification. Proc Natl Acad Sci U S A. 2009;106(3):835-40.

41. Ishikawa S, Maekawa M, Arite T, Onishi K, Takamure I, Kyozuka J. Suppression of tiller bud activity in tillering dwarf mutants of rice. Plant Cell Physiol. 2005;46(1):79-86.

42. Beveridge CA, Ross JJ, Murfet IC. Branching in pea (action of genes Rms3 and Rms4). Plant Physiol. 1996;110(3):859-65.

43. Kang C, Darwish O, Geretz A, Shahan R, Alkharouf N, Liu Z. Genome-scale transcriptomic insights into early-stage fruit development in woodland strawberry Fragaria vesca. Plant Cell. 2013;25(6):1960-78.

44. Hollender CA, Geretz AC, Slovin JP, Liu Z. Flower and early fruit development in a diploid strawberry Fragaria vesca. Planta. 2012;235(6): 1123-39.

45. Gu T, HanY, Huang R, McAvoy RJ, Li Y Identification and characterization of histone lysine methylation modifiers in Fragaria vesca Sci Rep, 2016;6:23581.

46. Gu T. RenS, WangY, HanY, Li Y. Characterization of DNA methyltransferase and demethylase genes in Fragaria vesca. Mol Gen Genomics. 2016;291(3): 1333-45.

47. Ruyter-Spira C, Al-Babili S, van der Krol S, Bouwmeester HJ. The biology of strigolactones. Trends Plant Sci. 2013;18(2):72-83.
48. Turnbull CG, Booker JP, Leyser O. Micrografting techniques for testing longdistance signalling in Arabidopsis. Plant J. 2002;32(2):255-62.

49. Bainbridge K, Sorefan K, Ward S, Leyser O. Hormonally controlled expression of the Arabidopsis MAX4 shoot branching regulatory gene. Plant J. 2005; 44(4):569-80.

50. Yoneyama $K$, Xie $X$, Kusumoto $D$, Sekimoto $H$, Sugimoto $Y$, Takeuchi $Y$, Yoneyama K. Nitrogen deficiency as well as phosphorus deficiency in sorghum promotes the production and exudation of 5-deoxystrigol, the host recognition signal for arbuscular mycorrhizal fungi and root parasites. Planta. 2007;227(1):125-32

51. Arite T, Umehara M, Ishikawa S, Hanada A, Maekawa M, Yamaguchi S, Kyozuka J. d14, a strigolactone-insensitive mutant of rice, shows an accelerated outgrowth of tillers. Plant Cell Physiol. 2009;50(8):1416-24.

52. Mouhu K, Kurokura T, Koskela EA, Albert VA, Elomaa P, Hytönen T. The Fragaria vesca homolog of suppressor of overexpression of constans 1 represses flowering and promotes vegetative growth. Plant Cell. 2013;25(9): 3296-310.

53. Ueda H, Kusaba M. Strigolactone regulates leaf senescence in concert with ethylene in Arabidopsis. Plant Physiol. 2015;169(1):138-47.

54. Lauressergues D, Andre O, Peng J, Wen J, Chen R, Ratet P, Tadege M, Mysore KS, Rochange SF. Strigolactones contribute to shoot elongation and to the formation of leaf margin serrations in Medicago truncatula R108. Exp Bot. 2015;66(5):1237-44.

55. Depuydt S, Hardtke CS. Hormone signalling crosstalk in plant growth regulation. Curr Biol. 2011;21(9):R365-73.

56. Cheng $Y$, Dai $X$, Zhao Y. Auxin biosynthesis by the YUCCA flavin monooxygenases controls the formation of floral organs and vascular tissues in Arabidopsis. Genes Dev. 2006;20(13):1790-9.

57. Cheng and Zhao, A role for auxin in flower development. J Integr Plant Biol. 2007:49(1):99-104.

58. Bartrina I, Otto E, Strnad M, Werner T, Schmülling T. Cytokinin regulates the activity of reproductive meristems, flower organ size, ovule formation, and thus seed yield in Arabidopsis thaliana. Plant Cell. 2011;23(1):69-80.

59. Cucinotta M, Manrique S, Guazzotti A, Quadrelli NE, Mendes MA, Benkova E, Colombo L. Cytokinin response factors integrate auxin and cytokinin pathways for female reproductive organ development. Development. 2016; 143(23):4419-24.

60. Yu H, Ito T, Zhao Y, Peng J, Kumar P, Meyerowitz EM. Floral homeotic genes are targets of gibberellin signaling in flower development. Proc Natl Acad Sci U S A. 2004;101(20):7827-32.

61. Yamaguchi N, Winter CM, Wu MF, Kanno Y, Yamaguchi A, Seo M, Wagner D. Gibberellin acts positively then negatively to control onset of flower formation in Arabidopsis. Science. 2014;344(6184):638-41.

62. Wang DH, Li F, Duan QH, Han T, Xu ZH, Bai SN. Ethylene perception is involved in female cucumber flower development. Plant J. 2010;61(5):862-72.

63. Liu L, Wang Z, Liu J, Liu F, Zhai R, Zhu C, Wang H, Ma F, Xu L. Histological, hormonal and transcriptomic reveal the changes upon gibberellin-induced parthenocarpy in pear fruit. Hortic Res. 2018;5:1.

64. Mariotti I, Picciarelli P, lombardi I, Ceccarelli N. Fruit-set and early fruit growth in tomato are associated with increases in indoleacetic acid, cytokinin, and bioactive gibberellin contents. J Plant Growth Regul. 2011; 30(4):405.

65. Nardozza S, Boldingh HL, Wohlers MW, Gleave AP, Luo Z, Costa G, MacRae EA, Clearwater MJ, Richardson AC. Exogenous cytokinin application to Actinidia chinensis var deliciosa 'Hayward' fruit promotes fruit expansion through water uptake. Hortic Res. 2017:4:17043.

66. Zhang S, Shi Q, Albrecht U, Shatters RG Jr, Stange R, McCollum G, Zhang S, Fan C, Stover E. Comparative transcriptome analysis during early fruit development between three seedy citrus genotypes and their seedless mutants. Hortic Res. 2017:4:17041.

67. Chen S, Wang X, Zhang L, Lin S, Liu D, Wang Q, Cai S, El-Tanbouly R, Gan L, Wu H, Li Y. Identification and characterization of tomato gibberellin 2oxidases (GA2OXs) and effects of fruit-specific SIGA20X1 overexpression on fruit and seed growth and development. Hortic Res. 2016;3:16059.

68. Symons GM, Chua YJ, Ross JJ, Quittenden LJ, Davies NW, Reid JB. Hormonal changes during non-climacteric ripening in strawberry. J Exp Bot. 2012; 63(13):4741-50.

69. Estrada-Johnson E, Csukasi F, Pizarro CM, Vallarino JG, Kiryakova Y, Vioque A Brumos J, Medina-Escobar N, Botella MA, Alonso JM, et al. Transcriptomic analysis in strawberry fruits reveals active auxin biosynthesis and signaling in the ripe receptacle. Front Plant Sci. 2017;8:889. 
70. Nitsch JP. Growth and morphogenesis of the strawberry as related to auxin. Am J Bot. 1950;37:211-5.

71. Jia HF, Chai YM, Li CL, Lu D, Luo JJ, Qin L, Shen YY. Abscisic acid plays an important role in the regulation of strawberry fruit ripening. Plant Physiol. 2011;157(1):188-99.

72. Chai $Y$, Zhang $Q$, Tian $L$, Li $C L$, Xing $Y$, Qin L, Shen YY. Brassinosteroid is involved in strawberry fruit ripening. Plant Growth Regul. 2013;69(1):63-9.

73. Given NK, Venis MA, Gierson D. Hormonal regulation of ripening in the strawberry, a non-climacteric fruit. Planta. 1988;174(3):402-6.

74. Merchante C, Vallarino JG, Osorio S, Aragüez I, Villarreal N, Ariza MT, Martínez GA, Medina-Escobar N, Civello MP, Fernie AR, et al. Ethylene is involved in strawberry fruit ripening in an organ-specific manner. J Exp Bot. 2013;64(14):4421-39.

75. Garrido-Bigotes A, Figueroa NE, Figueroa PM, Figueroa CR. Jasmonate signalling pathway in strawberry: genome-wide identification, molecular characterization and expression of JAZs and MYCs during fruit development and ripening. PLoS One. 2018;13(5):e0197118.

76. Cheng X, Ruyter-Spira C, Bouwmeester H. The interaction between strigolactones and other plant hormones in the regulation of plant development. Front Plant Sci. 2013;4:199.

77. Keane TM, Creevey CJ, Pentony MM, Naughton TJ, Mclnerney JO. Assessment of methods for amino acid matrix selection and their use on empirical data shows that ad hoc assumptions for choice of matrix are not justified. BMC Evol Biol. 2006;6:29.

78. Guindon S, Gascuel O. A simple, fast, and accurate algorithm to estimate large phylogenies by maximum likelihood. Syst Biol. 2003;52(5):696-704

79. Bailey $T L$, Elkan C. Fitting a mixture model by expectation maximization to discover motifs in biopolymers. Proc Int Conf Intell Syst Mol Biol. 1994;2:28-36.

80. Yu D, Tang H, Zhang Y, Du Z, Yu H, Chen Q. Comparison and improvement of different methods of RNA isolation from strawberry (Fragria $\times$ ananassa). J Agric Sci. 2012;4:51-6.

81. Amil-Ruiz F, Garrido-Gala J, Blanco-Portales R, Folta KM, Muñoz-Blanco J, Caballero JL. Identification and validation of reference genes for transcript normalization in strawberry (Fragaria $\times$ ananassa) defense responses. PLoS One. 2013;8(8):e70603.

82. Liu S, Chen W, Qu L, Gai Y, Jiang X. Simultaneous determination of 24 or more acidic and alkaline phytohormones in femtomole quantities of plant tissues by high-performance liquid chromatography-electrospray ionizationion trap mass spectrometry. Anal Bioanal Chem. 2013:405(4):1257-66.

83. Chen W, Gai Y, Liu S, Wang R, Jiang X. Quantitative analysis of cytokinins in plants by high performance liquid chromatography: electronspray ionization ion trap mass spectrometry. J Integr Plant Biol. 2010;52(10):925-32.

84. Liu S, Chen W, Fang K, Jiang X, Gai Y. Classification and characterization of unknown cytokinins into essential types by in-source collision-induced dissociation electrospray ionization ion trap mass spectrometry. Rapid Commun Mass Spectrom. 2012;26(17):2075-82.

85. Guo J, Han Y, Liu K, Sun P. Synthesis of the strigolactone analogues of GR24. Journal of Jinan University (Natural Science \& Medicine Edition). 2015;36(5): 374-7 (In Chinese).

\section{Ready to submit your research? Choose BMC and benefit from:}

- fast, convenient online submission

- thorough peer review by experienced researchers in your field

- rapid publication on acceptance

- support for research data, including large and complex data types

- gold Open Access which fosters wider collaboration and increased citations

- maximum visibility for your research: over $100 \mathrm{M}$ website views per year

At $\mathrm{BMC}$, research is always in progress.

Learn more biomedcentral.com/submissions 